FRED E. SCHRADER

\title{
KARL MARXENS SMITHKOMMENTAR VON 1861/62 IM HEFT VII
}

ZUR RATIONALITÄT DES APORETISCHEN ARBEITSBEGRIFFS

Der im folgenden erstmals vollständig publizierte Kommentar Marxens ${ }^{1}$ dokumentiert eine Wende in seiner Beurteilung der Arbeitswerttheorie Adam Smiths. Diese ist ihm seit dem Beginn seiner Beschäftigung mit der politischen Ökonomie präsent und provoziert ihn zu wiederholten Selbstverständigungen. Dabei steht das Verhältnis zwischen Smith und Ricardo im Mittelpunkt. Nach der prinzipiellen Übernahme der Arbeitswerttheorie läßt sich Marx zunächst von der Argumentation Ricardos gegen Smith leiten. Erst im vorliegenden Smithkommentar löst er sich von dieser Beurteilung und versucht, eine zentrale Problemstellung Smiths, die von Ricardo ausgeblendet wird, immanent nachzuvollziehen und begrifflich weiterzuentwickeln. Dies wird in das Manuskript von 1861-63 übernommen und findet schließlich im Kapital von 1867 seinen systematischen Ort in der Abhandlung des ,Lohnfetischismus”.

Marxens erste Rezeption Smiths und Ricardos, die sich in den 1844er Exzerpten aus den Hauptwerken beider Autoren niederschlägt, ${ }^{2}$ vollzieht sich unter dem Vorzeichen einer vehementen Ablehnung der Arbeitswerttheorie. Wie vor ihm Engels in den „Umrissen zu einer Kritik der Nationalökonomie"3 denunziert Marx die Bestimmung des Werts durch die Arbeit in methodischer Anlehnung an Feuerbach als eine unwahre Abstraktion von der Wirklichkeit. ${ }^{4}$ Noch in der Skizze „Reflection” von

1 Marx-Engels-Nachlaß B 91 A, Internationaal Instituut voor Sociale Geschiedenis.

2 Ebd., B 20, 23-24; vgl. K. Marx, F. Engels, Historisch-kritische Gesamtausgabe, Abt. I, Bd 3, Berlin 1932, S. 457ff.

3 F. Engels, ,Umrisse zu einer Kritik der Nationalökonomie”, in: Deutsch-Französische Jahrbücher, Paris 1844; K. Marx, F. Engels, Werke, Berlin 1956-68 (weiter MEW), Bd 1, S. 499ff.; 13., überarbeitete Aufl., Berlin 1981, S. 505ff.

4 Marx, Ricardo-Exzerpt, Marx-Engels-Nachlaß B 23-24; Historisch-kritische Gesamtausgabe, Abt. I, Bd 3, hier S. 494. 
1851 vertritt er en passant die These vom Veräußerungsprofit. ${ }^{5}$ Im selben Zeitraum zeichnet sich aber bereits ein Umdenken Marxens ab. Das Heft „bullion. Das vollendete Geldsystem”, worin er seine bisherigen Exzerpte zur Geld- und Zirkulationstheorie unter systematischen Gesichtspunkten ordnet, beginnt er interessanterweise mit einem Kommentar zu Smith, und obwohl dort nicht die Wertheorie, sondern die Funktionsbestimmungen des Geldes im Vordergrund stehen, behandelt Marx in diesem Kommentar doch der Sache nach das, was er später Smiths Entwicklung des Begriffs der abstrakten Arbeit nennt.

I.) Adam Smith.

Aus der Theilung der Arbeit geht hervor, daß Einer von der Waare des Andern abhängt, d.h. die Nothwendigkeit des Austauschs; jeder bedarf ausser seinem speziellen (besondern) Produkt seiner eignen Industrie eine[r] bestimmte[n] Quantität einer allgemein acceptirbaren Waare; wogegen er völlig den Ueberfluß seiner besondern Industrie austauscht. Verschiedne Materien successiv hierzu angewandt, schließlich Silber und Gold. Anfangs als Barren ohne Stempel und Zeichen, nach dem Gewicht. Später gestempelt, gemünzt. (A.S. p. 6.)

$\mathrm{Zu}$ bemerken: 1) Das Gold und Silber als allgemeine Waare, als das allgemeine Product jeder besondern Industrie, die Verkörperung, der Körper der Austauschbarkeit des Products, seiner Tauschfähigkeit. (Das Geld hier nur als Tauschmittel.) Verkörperter Tauschwerth des Products. Erster Prozeß nach A.S.: Trennung des Goldes und Silbers, des Geldes als des allgemeinen Products jeder Industrie von ihrem besondern Product. Aber Gold und Silber hier nur nach der Form des allgemeinen Aequivalents, des allgemeinen Pfands, da es selbst Waare ist und eine valeur intrinsèque besitzt. Gesellschaftliches Product. ${ }^{6}$

Wenig später, im Heft VIII von 1851, akzeptiert Marx schließlich in seinen Kommentaren zu Ricardos Principles of Political Economy die Arbeitswerttheorie. ${ }^{7}$

Weiter zeigt sich, daß Marx bis Zur Kritik der politischen Ökonomie von $1859^{8}$ mit der Übernahme der Arbeitswerttheorie zunächst auch Ricardos Sicht der Wert- und Profittheorie Smiths übernimmt, wonach dieser die

${ }^{5}$ Marx-Engels-Nachlaß B 54, abgedruckt in K. Marx, F. Engels, Gesamtausgabe (weiter MEGA), Erste Abteilung, Bd 10, Berlin 1978, S. 503, Z. 24.

6 Marx-Engels-Nachlaß B 81; vgl. hierzu F. E. Schrader, Restauration und Revolution. Die Vorarbeiten zum „Kapital” von Karl Marx in seinen Studienheften 1850-1858, Hildesheim 1980, S. 71.

7 Marx-Engels-Nachlaß B 55, abgedruckt in K. Marx, Grundrisse der Kritik der politischen Ökonomie (Rohentwurf) 1857-1858. Anhang 1850-1858, 2 Tle, Moskau 1939-41, Reprint Berlin 1953 (weiter Grundrisse), S. $787 \mathrm{ff}$.

8 MEGA, Zweite Abteilung (im folgenden wird nur auf diese Abteilung verwiesen), Bd 1, T. 1, Berlin 1976, S. 245f.; Grundrisse, S. 236; K. Marx, Zur Kritik der politischen Oekonomie. Erstes Heft, Berlin 1859, in MEW, Bd 13, S. 45. 
Bestimmung des Werts durch die Arbeit zwar zuerst entwickelt, dann aber wieder aufgegeben habe, und zwar durch die Einführung eines zweiten Wertmaßes, des Getreides. Den Kern dieses vermeintlichen Widerspruchs glaubt Ricardo gefunden zu haben, wenn er mit Bezug auf Smith gleich zu Beginn der Principles schreibt:

if the reward of the labourer were always in proportion to what he produced, the quantity of labour bestowed on a commodity, and the quantity of labour which that commodity would purchase, would be equal, and either might accurately measure the variations of other things: but they are not equal; the first is under many circumstances an invariable standard, indicating correctly the variations of other things; the latter is subject to as many fluctuations as the commodities compared with it.

Mit Getreide aber habe Smith ein nicht minder wertveränderliches Wertmaß gewählt als es Gold und Silber sind.$^{9}$ Diese Passage nahm Marx in sein Heft VIII von 1851 paraphrasierend in zwei Varianten auf. $\mathrm{Zu}$ Beginn des Ricardokommentars heißt es:

Der Wert der Arbeit und die Quantität Waren, die eine bestimmte Quantität Arbeit kaufen kann, sind nicht identisch. Denn das Produkt des Arbeiters oder ein Äquivalent dieses Produkts ist nicht = der Belohnung des Arbeiters. Also ist der Wert der Arbeit nicht das Maß der Werte, wie die auf die Quantität der Waren verwendete Arbeit. (p. 5) ${ }^{10}$

Unter der Überschrift „Unterschied zwischen Arbeitslohn und der auf die Produktion einer Ware verwandten Arbeit" exzerpiert Marx weiter hinten im Heft dieselbe Stelle noch einmal wie folgt:

Wenn die Belohnung des Arbeiters stets im Verhältnis wäre zu seinem Produkt, würde die Quantität der Arbeit bestowed on a commodity und die Quantität Arbeit, die diese Arbeit kaufen könnte, gleich sein, ... aber die letztre ist so vielen fluctuations unterworfen, wie die Ware selbst, womit sie verglichen wird. (p. 5) ${ }^{11}$

Beide Zitate, von denen das erste mit der Vorlage recht frei umgeht und das zweite sich unter Ausklammerung eines Textteils eng an Ricardo hält, wertet Marx im weiteren Verlauf seiner Arbeiten unterschiedlich aus. Zwar wird Ricardo in Zur Kritik als der wissenschaftlich strenge Arbeitswerttheoretiker vorgestellt, ${ }^{12}$ Smith hingegen ,,verwechselt beständig die Bestimmung des Werts der Waren durch die in ihnen enthaltene Arbeitszeit

9 D. Ricardo, On the principles of political economy and taxation, London 1821, S. 5.

10 Grundrisse, S. 787.

11 Ebd., S. 816.

12 MEW, Bd 13, S. 45. 
mit der Bestimmung ihrer Werte durch den Wert der Arbeit".13 Aber zugleich gelangt Marx ansatzweise zu einer differenzierteren Einschätzung der Position Smiths. Im Rohentwurf von 1857-58 heißt es:

Daß von dem Standpunkt der blosen Exchanges aus Salair des Arbeiters $=$ dem Werth des Products sein müßte, d.h. das Quantum Arbeit in objektiver Form, das der Arbeiter im Salair erhält, = dem Quantum Arbeit in subjektiver Form, das er in der Arbeit ausgiebt, ist so sehr nothwendige Consequenz daß A. Smith in sie verfällt. Ricardo hält dagegen am Richtigen fest, aber wie? „Der Werth der Arbeit und die Quantität Waaren, die eine bestimmte Quantität Arbeit kaufen kann, sind nicht identisch." Why not? „Denn das Product des Arbeiters oder ein Equivalent dieses Products ist nicht $=$ der Belohnung des Arbeiters." D.h. die Identität existirt nicht, weil der Unterschied existirt. ,Also (weil dem nicht so ist) ist der Werth der Arbeit nicht das Maaß des Werths, wie die auf die Quantität der Waaren verwandte Arbeit." $(19,5$.) Werth der Arbeit ist nicht identisch mit Belohnung der Arbeit. Denn sie sind verschieden. Also sind sie nicht identisch. $\mathrm{Die} ß$ ist ein curioser Schluß. Au fond liegt dem nichts zu Grunde, als daß es in der Praxis nicht so ist. Der Theorie aber nach müßte es so sein. ${ }^{14}$

Marx bezieht sich hier nicht auf den Text Ricardos, sondern auf den ersten seiner beiden paraphrasierenden Auszüge. Vergleicht man diesen mit der Vorlage, so zeigt sich gleich auf den ersten Blick, daß die bemängelte Argumentationslogik nicht von Ricardo, sondern von Marx selbst stammt. Aus ,but" wurde bei ihm ein ,denn”, und das konkludierende ,also" findet sich bei Ricardo nicht. Wenn Marx sich somit über eine merkwürdige Art der Beweisführung mokiert, dann richtet sich das sachlich zuallererst gegen Marxens freien Umgang mit seiner Vorlage und insbesondere gegen seine projektive Übersetzung. Ironischerweise trifft er aber durch dieses Selbstmißverständnis am Schluß seiner Ricardokritik richtig einen wunden Punkt in Ricardos Smithschelte, daß nämlich ,der Theorie nach” der Wert der Arbeit als Resultat des Arbeitsprozesses mit dem Arbeitslohn identisch sein müßte, es aber in der gesellschaftlichen Wirklichkeit nicht ist.

Daß auf der Basis der Arbeitswerttheorie der Wert der Arbeit als Resultat des Arbeitsprozesses mit dem Lohn identisch sein müßte, ist keine willkürliche Extrapolation Marxens. Er kann nämlich in diesem $\mathrm{Zu}$ sammenhang auf andere Autoren, hier auf John Francis Bray verweisen: „Bray z.B. glaubt daher mit seinem equal exchange zwischen lebendiger und todter Arbeit erst die wahre Consequenz aus Ricardo zu ziehn." 15

13 Ebd., S. 44f.

14 MEGA, Bd 1, T. 2, Berlin 1981, S. 455f.; Grundrisse, S. $455 f$.

15 Ebd., S. 455. 
Hinter Bray stehen Thomas Hodgskin, William Thompson, Piercy Ravenstone und andere, z.T. anonyme und pseudonyme Theoretiker der frühen englischen Arbeiterbewegung, ${ }^{16}$ aber auch Praktiker der Bewegung der ,Equal Labour Exchanges” in den 1820er und 1830er Jahren. ${ }^{17}$ Sie alle hängen Vorstellungen an, welche weiterhin von Intellektuellen der sozialen Bewegung wie Proudhon verbreitet wurden und sich noch in saint-simonistischen Derivaten bonapartistischer Bankprojekte wie denen der Brüder Péreire wiederfanden. ${ }^{18}$ Marx ist offensichtlich der Überzeugung, daß Proudhon seine englischen Vorläufer plagiiere, diese wiederum auf Ricardo aufbauten und eine radikale sozialkritische Interpretation seiner Theorie lieferten. ${ }^{19}$ Bei näherer Betrachtung erweist sich diese Auffassung nur als bedingt richtig. Denn jene Autoren greifen noch hinter Ricardo auf naturrechtliche Argumente zurück, die sich insbesondere bei Smith finden. Es handelt sich dabei um Konstruktionen eines geschichtlich-ursprünglichen oder vernünftig-natürlichen Gesellschaftszustands, worin die persönliche Arbeit ein Privateigentumsrecht am Arbeitsprodukt herstellt. ${ }^{20}$

Genau in diesem Zusammenhang, nämlich in der Auseinandersetzung mit Bray, Proudhon und ,den Saint-Simonisten", 21 nimmt Marx ein weiteres Moment des Einwandes Ricardos gegen Smith wieder auf, welches

16 J. F. Bray, Labour's wrongs and Labour's remedy; or the Age of Might and the Age of Right, Leeds 1839; Th. Hodgskin, Popular political economy. Four lectures delivered at the London Mechanics' Institution, London 1827; [ders.,] Labour defended against the claims of capital; or, the unproductiveness of capital proved with reference to the present combinations amongst journeymen. By a labourer, London 1825; W. Thompson, An inquiry in to the principles of the distribution of wealth most conducive to human happiness: applied to the newly proposed system of voluntary equality of wealth, London 1824; P. Ravenstone, A few doubts as to the correctness of some opinions generally entertained on the subject of population and political economy, London 1821; The source and remedy of the national difficulties, deduced from principles of political economy in a letter to Lord John Russell, London 1821.

$17 \mathrm{Vgl}$. deren Berichte in The Crisis; or the Change from Error and Misery, to Truth and Happiness, hrsg. von R. Owen, 1832, und The Poor Man's Guardian. Established, contrary to ..Law”, to try the Power of "Might” against „Right”, 1831-35.

18 Isaak Péreire, in seiner Jugend Saint-Simonist und Anhänger Enfantins, gründete 1852 mit seinem Bruder Emile und anderen den Crédit mobilier, von Marx als Institution des „kaiserlichen Sozialismus” bekämpft; vgl. Marxens Artikel in der New-York Daily Tribune von 1856, MEW, Bd 12, S. $20 \mathrm{ff}$.

19 K. Marx, Misère de la philosophie. Réponse à la philosophie de la misère de $\mathrm{M}$. Proudhon, Paris, Brüssel 1847, in Historisch-kritische Gesamtausgabe, Abt. I, Bd 6, Berlin 1932, S. 150; vgl. MEW, Bd 4, S. 98.

$20 \mathrm{Vgl}$. den Brief Hodgskins an Place vom 28. Mai 1820, in: E. Halévy, Thomas Hodgskin (1787-1869), Paris 1903, S. 54ff. und auch S. 123. Marx sieht die Verbindung, s. unten, S. 71 .

21 MEGA. Bd 1, T. 1, S. 89: Grundrisse, S. 73. 
sich im zweiten der beiden Auszüge der Seite 5 der Principles findet. Demnach kann die Arbeit selbst nicht unmittelbares Wertmaß des Arbeitsprodukts sein. Auch wenn der Preis nämlich seine Denomination in der Arbeit erhielte, wäre er laufenden Schwankungen unterworfen. Deshalb muß als vorgestellte Geldmaterie eine andere Ware verwandt werden. ${ }^{22}$

Gegen Smith führt Ricardos Verweis auf die Differenz zwischen Wert und Preis allein nicht sehr weit. Darum wirft Marx ihm Begrifflosigkeit gegenüber dem Problem vor, das Smith beschäftigt: dem eklatanten Widerspruch zwischen Theorie und Praxis der Wertbestimmung durch die Arbeit. Darüber hinaus aber führt Ricardos Kritik an Smith regelrecht in die Irre, und zwar auch Marx. Denn Smith gibt die Arbeitswerttheorie durchaus nicht auf, wie Ricardo im ersten und achtundzwanzigsten Kapitel der Principles behauptet. ${ }^{23}$ Vielmehr schlägt Smith Getreide, dessen Wert er für vergleichsweise konstant hält, als theoretisches langfristiges Maß der Werte - nicht als Wertsubstanz - statt der seines Erachtens im Wert stärker schwankenden Edelmetalle vor. Hätte er die Konstruktion einer anderen Werttheorie vor Augen, wären seine durchgängig eingeworfenen ausdrücklichen Hinweise unerklärlich, wonach bei allen seinen Überlegungen selbstverständlich die Arbeit Maß und Substanz des Werts bleibe. ${ }^{24}$ Ricardos ebenso fehlleitende wie selbstbegünstigende Behauptung, Smith erhebe das Getreide zum vermeintlich wahren Wertmaß, haben Sozialisten wie Thomas Rowe Edmonds wiederum positiv aufgegriffen und in der englischen Arbeiterbewegung gegen das herrschende „Geldsystem” eine Währung mit Getreidestandard zum Aufbau einer neuen Sozialordnung propagiert, ${ }^{25}$ was Marx bereits 1845 zur Kenntnis nimmt und 1851 erneut diskutiert. ${ }^{26}$

Wenn Marx zunächst auch unter starkem Einfluß der selbstbegünstigenden Argumentationsstrategie Ricardos steht, so wirft er ihm doch bereits im Rohentwurf ein Unverständnis der Problemstellung Smiths

22 MEGA, Bd 1, T. 1, S. $71 \mathrm{ff}$; Grundrisse, S. $55 \mathrm{ff}$.

23 D. Ricardo, Grundsätze der politischen Ökonomie und der Besteuerung, Frankfurt/ M. 1972 , S. $276 \mathrm{ff}$.

24 A. Smith, Eine Untersuchung über Wesen und Ursachen des Volkswohlstandes, Jena 1923 (Reprint Gießen 1973), Buch I, „Besondere Abhandlung über die Veränderungen im Silberwerte während der letzten vier Jahrhunderte”, S. 251-58 und 267-70.

25 T. R. Edmonds, Practical moral and political economy; or, the government, religion, and institutions, most conducive to individual happiness and to national power, London 1828, S. 283-98; dies wird nicht erwähnt in E. Nolte, „Thomas Hopkins und T. R. Edmonds - zwei vergessene ,ricardische Sozialisten”? Zugleich ein Beitrag zur Begriffsklärung", in: International Review of Social History, Jg. 26 (1981), S. 77ff.

${ }^{26}$ Marx-Engels-Nachlaß B 29 und 81; vgl. Schrader, Restauration und Revolution, a.a.O., S. $55 \mathrm{ff} ., 78 \mathrm{ff}$. 
gegenüber vor. Drei Jahre später versucht er im vorliegenden Kommentar erstmals, Smiths Gedankenführung immanent nachzuvollziehen. Ausgangspunkt der Überlegungen ist ein unentwickelter Zustand der Gesellschaft, für den Smith annimmt, daß die persönliche Arbeit das Privateigentum am Arbeitsprodukt begründet und dessen Wert von jener Arbeit bestimmt wird. Den Wendepunkt solcher ,antehistorischer Zeiten”, wie Marx sie nennt, führt Smith mit der Kapitalakkumulation, dem Privateigentum an Grund und Boden, der Produktivitätssteigerung der Arbeit und der allgemeinen Eigentumslosigkeit der Arbeiter an den Produktionsbedingungen ein. ${ }^{27}$ Wie Marx bereits im Rohentwurf anmerkt, treten Kapitalist und Grundeigentümer bei Smith außerhalb der ökonomischen Logik ,als historische facts mit ihren Ansprüchen" 28 auf und erzwingen diese mit zunächst außerökonomischen Mitteln wie beispielweise besonderen Gesetzen, die dann aber selbst zu allgemeinen Produktionsbedingungen werden. ${ }^{29}$ Die Resultate des geschichtlichen Einbruchs beschreibt Smith nach Marx in mehrfacher Weise: Die Arbeit bestimmt zwar weiterhin ökonomisch eindeutig den Wert des Arbeitsprodukts, aber die naturrechtliche Verknüpfung zwischen persönlicher Arbeit und Privateigentum an deren Produkt ist aufgelöst; das (Wert-)Produkt des Arbeiters teilt sich nunmehr in Lohn, Profit und Rente; dadurch schließlich ist die gesellschaftlich durchschnittliche Arbeitsquantität, mit der man eine Ware erhält oder produziert, nicht mehr das einzige, was die Arbeitsquantität bestimmt, welche diese Ware kaufen, austauschen oder ,kommandieren” kann. Denn, so heißt es bei Smith weiter, es ist nun noch eine zusätzliche Quantität für den Profit des Kapitals fällig, das den Lohn und die Produktionsbedingungen vorgeschossen hat.

Marx versucht an dieser Stelle, Smiths Gedankengang wie folgt zu rekonstruieren:

Also: weil die in der Waare enthaltene Arbeit nicht mehr $=$ der Arbeit, die mit der Waare gekauft werden kann [...], hört die in der Waare enthaltne Arbeit auf Maaß des Werths für A. Smith zu sein. Dagegen Ricardo. Oder Smith glaubt daß dieß Werthmaaß aufgehört hat, weil es prima facie nicht anwendbar ist auf das Verhältniß von Capital und Lohnarbeit. Er nimmt daher als Maaß der Werthe (was er jedoch nirgend durchführen kann, weil es in sich absurd ist) das Quantum Arbeit, das ich mit einer Masse Waaren kaufen kann, oder die Masse Waaren, die ein bestimmtes Quantum Arbeit erstehn kann, ein Quantum was ganz von der Grösse des Salairs abhängt $[\ldots .]^{30}$

${ }^{27}$ MEGA, Bd I, T. 1, S. 89; Grundrisse, S. 74, vgl. S. 904.

${ }^{28}$ MEGA, Bd 1, T. 1, S. 245; Grundrisse, S. 236.

${ }^{29}$ MEGA, Bd 1. T. 2, S. 416; Grundrisse, S. 413.

30 Unten S. 76. 
Bemerkenswert ist, daß Marx selbst schreibt, Smith führe seinen neuen Ansatz nirgendwo durch, weil er in sich absurd sei. Denn auf der anderen Seite nimmt Marx durchaus die Behauptung Ricardos auf, Smith führe als vermeintlich wahres Wertmaß im elften Kapitel des ersten Buchs der Inquiry das Getreide ein, und versucht, dies mit der Arbeitswerttheorie Smiths zu einem einigermaßen stimmigen Bild zusammenzubringen: Smith nehme die Arbeit, für die das Getreide als Gebrauchswert des Arbeitslohns eingetauscht wird, als konstant an und mache sie zum Maß aller Werte. ${ }^{31}$ Offensichtlich versucht Marx mit diesem Satz eine mögliche Intention Smiths zu treffen, ist sich aber dessen bewußt, daß dieser selbst sich eine solche Entwicklung seiner Theorie verwehrt hat, weil dies zu inneren Widersprüchen geführt hätte.

An der Arbeitswerttheorie hält Ricardo nach eigener Darstellung konsequenter fest als Smith. Marx wiederum wirft Ricardo vor, er blende anders als Smith - die Darstellung des Verhältnisses von Lohnarbeit und Kapital auf der Basis der Arbeitswerttheorie aus. Unter dem Eindruck der ,linksricardianischen” Argumentationswelle in der frühen englischen Arbeiterbewegung, die ganz ungeniert Smiths Begründung des Profits und der Rente durch außerökonomischen Zwang und eine Gesetzgebung wider das Naturrecht gegen Ricardo ausspielte, hat die politische Ökonomie diese Leerstelle bei Ricardo gefüllt, indem sie mit Blick auf die Legitimation des Profits die Funktion des Arbeitswerts drastisch reduzierte - und damit auch die Legitimation der Aneignung des (Wert-)Produkts durch persönliche Arbeit. Den eminent politischen Anlaß für diese theo-

31 Die ,Selbstverständigung" findet sich im Heft XII des Manuskripts von 1861-63: „Und hier haben wir den verborgnen Grund, warum A. Smith sagt, daß sobald das Capital dazwischen kommt und folglich die Lohnarbeit, nicht die quantity of labour bestowed upon the produce, but the quantity of labour it can command, seinen Werth regulirt. Der durch Arbeitszeit bestimmte Werth des Korns (and of other necessaries) wechselt; aber, solange der natural price of labour bezahlt wird, bleibt das Quantum Arbeit, das das qr Korn commandirt dasselbe. Es hat also einen permanenten relativen Werth, verglichen mit Korn. Darum auch bei Smith, value of labour und value of corn (for food. Sieh D. Hume) standard measures of value [...]. Sagt Ric: Das qr corn hat sehr verschiednen Werth, obgleich es stets dasselbe Quantum Arbeit commandirt oder davon commandirt wird. Ja, sagt A. Smith: Wie immer der durch Arbeitszeit bestimmte Werth des qr Korn wechsle, der Arbeiter muß stets dasselbe Quantum Arbeit zahlen (Opfer bringen), um es zu kaufen. Also wechselt der Werth des Korns, aber der Werth der Arbeit wechselt nicht, denn I Monat Arbeit $=1 \mathrm{qr}$ Korn. Auch der Werth des Korns wechselt nur, sofern wir die Arbeit betrachten, die zu seiner Production erheischt ist. Beobachten wir dagegen das Quantum Arbeit, wogegen es sich austauscht, das es in Bewegung sezt, so wechselt sein Werth nicht. Und darum ist eben die quantity of labour, against which a qr of corn is exchanged, the standard measure of value." MEGA, Bd 3, T. 3, Berlin 1978, S. 1026f. Marx bezieht sich hier auf den Perspektivwechsel in Smith, Buch I, Kap. 5, vgl. A. Smith, Recherches sur la nature et les causes de la richesse des nations (vollständiger Titel in Anm. 58). S. 64-66, und Eine Untersuchung, a.a.O., S. 40-46. 
retische Anstrengung spricht Carey offen aus. ${ }^{32}$ Wakefield greift aus demselben Grund in einer Fußnote zu Smith und Ricardo faktisch dessen Smithkritik auf und will den Profit aus den durch das Spiel von Angebot und Nachfrage unterhaltenen Oszillationen des Preises und hier insbesondere des Lohnes entstehen lassen. ${ }^{33}$

Daß Ricardo selbst schon dieser Entwicklung Raum gegeben hat, erklärt Marx zur wissenschaftlichen Schwäche des Gesellschaftstheoretikers. Im theoriegeschichtlichen Teil des Manuskripts von 1861-63 verarbeitet er den Smithkommentar weiter und kann schließlich eine Bilanz ziehen, die sich von seinen alten Einschätzungen dadurch unterscheidet, daß er Smith explizit gegen Ricardos Fehlinterpretation verteidigt. Dieser bestimme den „Wert der Arbeit” nicht auf der Basis der Arbeitswerttheorie, sondern unter Verwendung des Gesetzes von Angebot und Nachfrage durch den Wert des Geldlohns und damit der Sache nach durch das Quantum Gebrauchswerte, welches mit diesem Lohn gekauft wird; wörtlich verfalle Ricardo auf diese Weise genau in die von ihm selbst Smith vorgehaltene Inkonsequenz. Außerdem aber wirft Marx Ricardo vor, er verstehe nicht einmal das Problem, mit dem sich Smith herumschlage. ${ }^{34}$

Als Lösung des Dilemmas Smiths und Ricardos erscheint seit dem Rohentwurf die begriffliche Differenzierung zwischen Arbeit und Arbeitsvermögen bzw. -kraft des Lohnarbeiters. ${ }^{35}$ Unter dieser Voraussetzung kann Marx die Arbeitswerttheorie beibehalten, auf ihrer Grundlage das Verhältnis zwischen Lohnarbeit und Kapital beschreiben und den „Wert der Arbeit” bestimmen durch ,die Arbeitsquantität bestowed upon the force of labour". ${ }^{36}$ Dementsprechend wird in der Theoriegeschichte

${ }^{32}$ H. C. Carey, The past, the present, and the future, Philadelphia 1848, S. $74 \mathrm{f}$.

33 E. G. Wakefield, Anm. zu Buch I, Kap. 8-9, in: A. Smith, An inquiry into the nature and causes of the wealth of nations, London 1843, Bd 1, S. 230f.

${ }_{34}$ MEGA, Bd 3, T. 3, S. 1027; vgl. aber Marxens Erläuterung im Kapital, MEW, Bd 23. S. 186: „Der Wert der Arbeitskraft löst sich auf in den Wert einer bestimmten Summe von Lebensmitteln. Er wechselt daher auch mit dem Wert dieser Lebensmittel. d.h. der Größe der zu ihrer Produktion erheischten Arbeitszeit." Daß Marx sich nicht völlig von der Ricardoschen Smithkritik löst, mag daran liegen, daß er diese implizit mit seiner eigenen Kritik an Smiths Darstellung des gleichbleibenden Werts der Arbeit interpoliert; vgl. Smith, Wealth of nations, Buch I, Kap. 5; ders., Eine Untersuchung, Bd 1, S. 40f.; in der Übersetzung Marxens, MEW, Bd 13, S. 45, Anm.: „Gleiche Quantitäten der Arbeit müssen zu allen Zeiten und an allen Orten für den, welcher arbeitet, einen gleichen Wert haben. In seinem normalen Zustand von Gesundheit, Kraft und Tätigkeit, und mit dem Durchschnittsgrad von Geschicklichkeit [. . .] muß er immer die nämliche Portion seiner Ruhe, Freiheit und seines Glücks geben. Welches also immer die Quantität von Waren sei, die er als Belohnung seiner Arbeit erhält, der Preis, den er zahlt, ist immer derselbe. [...] Die Arbeit allein wechselt also nie ihren eigenen Wert."

${ }_{35}$ Zuerst im Rohentwurf, MEGA. Bd 1, T. 1, S. 198ff.; Grundrisse, S. $185 \mathrm{ff}$.

36 MEGA, Bd 3, T. 3, S. 1027. 
Marxens wesentlicher Beitrag zu diesem Problem auch als Überwindung des Smith-Ricardoschen Begriffs des Werts der Arbeit dargestellt. Das ist jedoch noch eine Beschreibung des Problems und bleibt diesem selbst noch äußerlich. Darüber hinaus stellt sich die Frage, was das Problem selbst ausmacht, welches Smith vergeblich zu lösen versucht und Ricardo gar nicht erst zuläßt.

Das Problem Smiths taucht beim Übergang vom angenommenen ursprünglichen Zustand der Gesellschaft zu ihrem modernen auf. Im ersten begründet die persönliche Arbeit das Privateigentum am Arbeitsprodukt und ist dessen Wert mit jener Arbeit identisch. Als reine Quantitäten bilden sich Arbeit und Wert des Arbeitsprodukts gegenseitig eindeutig ab, wobei beide durch die Identität des Arbeiters als Aneigners miteinander verknüpft sind und sich diese Identität der Person mit der des Begriffs der Arbeit (als Prozeß und als Resultat) deckt. Mit der weiter von Smith angenommenen historischen Auflösung der Identität von Arbeiter und privatem Aneigner seines Arbeitsprodukts als Klassenscheidung auf gesamtgesellschaftlicher Ebene endet auch die begriffliche Identität der Arbeit. Smith will jedoch daran festhalten und versucht, sie über das Austauschverhältnis herzustellen. Er kommt dabei aber nur zu dem Satz, der Realwert von Arbeitslohn, Profit und Rente, woraus sich der Preis einer Ware zusammensetze, werde nach der Arbeitsquantität gemessen, welche jeder dieser Bestandteile kaufen oder sich verfügbar machen könne. In diesem Satz verwendet Smith nur einmal den Begriff der Arbeit als Quantität. Der Sache nach handelt es sich aber erstens um Arbeit in der Form des Resultats, zweitens um solche in der des Resultats und zugleich des möglichen Prozesses. Im zweiten Fall zeigt sich eine neue Doppeldeutigkeit des Arbeitsbegriffs, welche zu derjenigen hinzutritt, die vorher schon durch die begriffliche Einheit von ArbeitsprozeB und Arbeitsprodukt bestand. Smiths Schwierigkeit resultiert demnach aus der Mehrdeutigkeit des von ihm verwandten Arbeitsbegriffs.

Die Mehrdeutigkeit kommt genauer besehen dadurch zustande, daß Smith Wert und Arbeit sich genetisch und analytisch gegenseitig begründen läßt und die Arbeit hierbei wie den Wert als reine Quantität neben der realen Arbeit behandelt. Erst durch diesen Begriff der abstrakten Arbeit als negative Einheit aller konkreteren Bestimmungen des Arbeitsbegriffs bilden sich die Schwierigkeiten, mit denen Smith zu kämpfen hat. Auf der anderen Seite kann er aber auch nicht zu den konkreteren Bestimmungen flüchten, weil das ein Rückschritt wäre. Schließlich beruht seine systematische Leistung eben auf der Entwicklung des abstrakten Arbeitsbegriffs zu einer Werttheorie. Marx weist mehrmals darauf hin, daß Smith der erste Theoretiker ist, der einen Begriff der 
abstrakten Arbeit, der "Arbeit sans phrase" im Unterschied und im Gegensatz $\mathrm{zu}$ den vielen besonderen konkreten Arbeiten verwendet. ${ }^{37}$ Dem gibt Marx weiter eine spezifische geschichtliche Begründung. Es handele sich dabei nicht um eine vereinzelte intellektuelle Abstraktionsleistung des Wissenschaftlers Smith, sondern um eine Abstraktion auf der Grundlage der modernen bürgerlichen Gesellschaft, worin sich einerseits eine Totalität voneinander unterschiedener konkreter Arbeiten ausgebildet habe und andererseits die jeweils bestimmte Konkretion der Arbeit zufällig und gleichgültig sei: ,Hier also wird die Abstraction der Categorie ,Arbeit' [...], der Ausgangspunkt der modernen Oekonomie, erst praktisch wahr." 38 Auf dieser Grundlage schlüsselt Marx den Begriff der abstrakten Arbeit bei Smith als Substanz des abstrakten (Tausch-)Werts auf, des ,allgemeinen Produkts”, welches jeder Arbeiter mit und neben seinem besonderen Produkt erzeuge. ${ }^{39}$

Wenn in der modernen bürgerlichen Gesellschaft die Verwandlung aller Arbeitsprodukte in Produkte und Tauschwerte stattfindet, was sie als Waren konstituiert, dann handelt es sich dabei nicht um vereinzelte Abstraktionen, sondern um eine kollektive intellektuelle Leistung, die im Vergesellschaftungsproze $\beta$ von den verstreuten Individuen massenhaft und in prinzipiell unendlicher Wiederholung vorgenommen wird. Dadurch - und analog dazu über die Abstraktionsform des Privateigentums - stellen sie überhaupt erst ihren sachlichen gesellschaftlichen mitsamt ihrem privaten, persönlichen Reproduktionszusammenhang her. Dieser Zusammenhang, in dem die von Smith erstmals begrifflich rein dargestellte abstrakte Arbeit als Substanz des Werts auftritt, ist nach Marx die sachliche Grundlage dafür, daß in der Philosophie als Merkmal der „Neuen Zeit" die Herrschaft von Abstraktionen oder Ideen über die Individuen angesehen wurde. ${ }^{40}$

Hierauf aufbauend bemerkt Marx, daß der gesellschaftlich lückenlos fortwährend reproduzierte Abstraktionsprozeß mittels der Begriffsbildung der „Arbeit sans phrase" für sich allein genommen gar nicht zwingend notwendig ist und als ein sinnloser Prozeß erscheint. ${ }^{41}$ Vielmehr erscheinen ideelle und wirkliche Verdoppelung des Arbeitsprodukts in

${ }^{37}$ MEGA, Bd 1, T. 1, S. 39; Grundrisse, S. 24f.

38 Ebd.

${ }^{39}$ MEGA, Bd I, T. 1, S. 100f.; Grundrisse, S. 86. Wenn Smith daneben noch konkrete Vorstellungen von Arbeit als „Arbeit” behandelt, dann erklärt Marx dies mit dem relativ unentwickelten Zustand der bürgerlichen Gesellschaft im 18. Jahrhundert, vgl, auch MEGA, Bd I, T. 2, S. 498ff.; Grundrisse, S. 504ff.

${ }^{40}$ MEGA, Bd 1, T. 1, S. 96f;; Grundrisse, S. 81 f.

${ }^{41}$ MEGA, Bd 1, T. 1, S. 177; Grundrisse, S. 166. 
Ware, Tauschwert, Preis und Geld überhaupt nur unter der gesellschaftlich allgemeinen Voraussetzung des Austauschs zwischen Lohnarbeit und Kapital als sinnvoll. Somit erweist sich die gesellschaftliche Allgemeinheit des Austauschs von jeweils als Werte unter der abstrakt-rech tlichen Form des Privateigentums organisierten Produkten zur Funktion dieses theoretischen Sonderfalls des Austauschs. Seit dem Rohentwurf beschreibt Marx den Vorgang in Anlehnung an Cherbuliez als dialektischen Umschlag des Äquivalenttauschs, wobei er den Begriff des Werts des Arbeitsvermögens einführt. ${ }^{42}$ Die Mehrwertigkeit des Arbeitsbegriffs, welche Smith zum Problem wird, erscheint schließlich im Kapital als gesellschaftlich rationell. Auf der Basis seiner begrifflichen Differenzierung beschreibt Marx dies als „Lohnfetischismus”. Wenn sich im Bewußtsein insbesondere der Lohnarbeiter Arbeit(szeit), Wert sowie Arbeitsprodukt als Lohn der Arbeit zur begrifflichen Identität verschränken und sich gegenseitig eindeutig abbilden, dann löscht die Form des Geldlohns als Preis der geleisteten Arbeit jede Spur der von Marx so genannten Teilung des Arbeitstages in notwendige Arbeit und Mehrarbeit aus. ${ }^{43}$ Hierzu aber ist die Bildung des abstrakten Arbeitsbegriffs, und zwar durch die beteiligten empirischen Personen selbst, eine gesellschaftlich notwendige Voraussetzung.

Die Rationalität des abstrakten Arbeitsbegriffs liegt demnach außerhalb seiner selbst im gesellschaftlichen Reproduktionsverhältnis, das sich durch jene Begriffsbildung als einer seiner wesentlichen Formen vollzieht. Dieser Sachverhalt zwingt Marx schon im Rohentwurf zur Ausarbeitung einer eigenständigen Darstellungsdialektik in seiner Kritik der politischen Ökonomie. ${ }^{44} \mathrm{Da}$ selbst er noch im Kapital nicht umhin kann, weiterhin vom „Preis der Arbeit” zu schreiben, um das Verhältnis von Arbeit und Kapital samt dem ihm inhärenten Fetischismus abhandeln zu können, zeigt den unwillkürlich hegelsch anmutenden, doch inzwischen von Marx materialistisch begründeten Widerstand des gesellschaftlich rationellen Begriffs gegen seinen analytischen Zugriff an. ${ }^{45}$ Das Problem Smiths, auf

42 MEGA, Bd 1, T. 1, S. 365ff., 416f.; Grundrisse, S. 360ff., 413f.; Das Kapital, MEW, Bd 23, S. 184, Anm. 41, und S. 610, Anm. 23.

43 MEW, Bd 23, S. 559, 561ff.

44 Daraus erwächst entgegen Marxens Absicht seine ,idealistische Manier der Darstellung" in der Form einer Begriffsentwicklung. Vgl. Rohentwurf, MEGA, Bd 1, T. 1, S. 85; Grundrisse, S. 69.

45 Nachdem Marx die Geschichte der „Kategorie ,Preis der Arbeit”, die geradewegs zum Begriff „Wert der Arbeit” führte, dargestellt und weiterhin auseinandergesetzt hat, daß die Mystifikation gerade in ,der Verwandlung von Wert und Preis der Arbeitskraft in die Form des Arbeitslohns oder in Wert und Preis der Arbeit selbst" besteht, schreibt er weiterhin wiederholt zwar nicht vom Wert, wohl aber vom „Preis der Arbeit” als dem „Geldwert eines gegebnen Quantums Arbeit” oder „Preis der Arbeitsstunde”; gemeint ist hiermit der ,durchschnittliche Tageswert der Arbeitskraft [geteilt] durch die Stun- 
dessen Relevanz Marx gegenüber Ricardo bemerkenswert hartnäckig insistiert, erweist sich somit zusammengefaßt nicht als bloß immanent begriffsgeschichtliches, sondern über die reproduktive und zugleich legitimierende Funktion der massenhaft individuell vorgenommenen begrifflichen Abstraktionsformen von Arbeit und Privateigentum wesentlich als ein Problem der gesellschaftlichen Praxis.

Der Smithkommentar ist in dem Heft überliefert, welches Marx im Februar 1858 als Heft VII des Rohentwurfs von Zur Kritik der politischen Ökonomie begann. Dieses Manuskript endet auf der Heftseite 63 Marxscher Paginierung. Mit der Seite 63(a), die Marx auf den 25. Februar 1859 datierte, beginnt der umfangreiche Exzerptteil des Hefts, der auf der Seite 276 endet, gefolgt von einer Inhaltsangabe auf der Innenseite des hinteren Umschlagdeckels. Diesen Exzerptteil hat Marx während der Vorbereitung und der Ausarbeitung des dritten Kapitels von Zur Kritik geführt.

Zwar ist der Smithkommentar nicht datiert, doch läßt sich seine Entstehungszeit eingrenzen. Er beginnt mit den Seiten 173 und 174, wird dann durch andere Exzerpte unterbrochen, auf der Seite 186 wiederaufgenommen und endet vorerst etwa in der Mitte der Seite 192, um schließlich auf Seite 210 noch einmal aufgegriffen zu werden. Kurz vor dem Kommentar, auf den Seiten 157ff. und 166, finden sich Auszüge aus den Reports of the Inspectors of Factories (1857) und aus Engels' Lage der arbeitenden Klasse in England. ${ }^{46}$ Marx berichtete Engels am 11. Januar 1860, er habe dessen Buch gerade im British Museum mit den Reports verglichen. ${ }^{47}$ Der Smithkommentar dürfte nach diesem Datum entstanden sein. Wahrscheinlich ist die Abfassung ein bis zwei Jahre später anzusetzen. Marx benutzte zunächst nicht wie üblich eine englische Smithausgabe, ${ }^{48}$ sondern die französische Übersetzung Germain Garniers von 1802, aus der er schon 1844 exzerpiert hatte ${ }^{49}$ und die er mit seiner Bibliothek 1849 in Köln

denzahl des durchschnittlichen Arbeitstags”, vgl. MEW, Bd 23, S. 562, 565f. und passim. Nicht nur im ,Wert der Arbeit”, auch im „Preis der Arbeit” bzw. „Preis der Arbeitsstunde" ist der Unterschied zwischen bezahlter notwendiger Arbeit und unbezahlter Mehrarbeit ausgelöscht.

${ }^{46}$ Reports of the Inspectors of Factories to Her Majesty's Principal Secretary of State for the Home Department, 4 Bde, London 1857-58; F. Engels, Die Lage der arbeitenden Klasse in England. Nach eigner Anschauung und authentischen Quellen, Leipzig 1845, in MEW, Bd 2, S. $225 \mathrm{ff}$.

47 MEW, Bd 30, S. 7 .

48 Wenn Marx sich nicht zur privaten Selbstverständigung seiner Smithexzerpte von 1844 bediente, dann bevorzugte er schon in seinen ersten Londoner Exzerptheften eine englische Ausgabe Smiths, so im Heft VII von 1851, Marx-Engels-Nachlaß B 54.

49 Smith, Recherches sur la nature et les causes de la richesse des nations, a.a.O. 
hatte zurücklassen müssen. ${ }^{50}$ Die Bibliothek wurde Marx 1860 nachgesandt und traf im Dezember des Jahres in London ein. Erst im Februar 1861 konnte Marx über ihre Freigabe berichten,${ }^{51}$ d.h. also kurz vor seiner Reise nach Holland und Deutschland, von wo er Ende April zurückkehrte. In einem Brief an Engels vom 10. Juni endlich schrieb Marx, er habe seit einer Woche ernsthaft mit seinem Buche begonnen. ${ }^{52}$ Das wird sich auf Vorarbeiten beziehen, denn das Heft I des Manuskripts selbst datierte er auf August 1861. Als unterste Grenze für die Entstehung des Smithkommentars ist demnach der Juni 1861 anzunehmen. Wegen seiner Nähe zum Manuskript von 1861-63 ist aber eine spätere Abfassung plausibel. Die Seite 193 des Exzerpthefts benutzte Marx für einen Auszug aus The Standard vom 25. Februar 1862. Die Exzerpte auf den Seiten 207 und 208 aus Watson, dem Bengal Hurkaru und dem Bericht der Bombay Chamber of Commerce ${ }^{53}$ hat er am Schluß des Hefts V des Manuskripts von 1861-63 aufgenommen, ${ }^{54}$ offenbar nach dem 25 . Februar 1862. Dafür spricht, daß der Brief Marxens an Engels vom 6. März 1862 sich auf eine ebenfalls im Heft $\mathrm{V}$ behandelte Frage bezieht. ${ }^{55}$ Demnach wäre - unter der Voraussetzung einer chronologischen Reihenfolge der Hefteinträge - der Smithkommentar spätestens Anfang März 1862 vor dem Abschnitt über Smith im Heft VI des Manuskripts von 1861-63 entstanden. ${ }^{56}$

Ein unmittelbarer Anlaß für die Niederschrift des Smithkommentars ist nicht zu erkennen. Vieles spricht dafür, daß er als erster Entwurf zur Vorbereitung des entsprechenden Kapitels über Smith im Heft VI entstanden ist, indem Marx aus seiner alten eigenen französischen Ausgabe die ihm relevant erscheinenden Passagen zusammengetragen und strukturiert hat, um dann ergänzend auf die englische, von John Ramsay MacCulloch besorgte Smithedition zurückzugreifen. ${ }^{57}$ Der gesamte Kommentar ist schließlich in das Manuskript von 1861-63 eingearbeitet worden.

Marxens Smithkommentar scheint keinem besonderen Plan zu folgen.

50 Jedenfalls weist Daniels’ Bestandsverzeichnis von 1850 diesen Titel auf; vgl. Ex libris Karl Marx und Friedrich Engels. Schicksal und Verzeichnis einer Bibliothek, Berlin 1967, S. 213.

51 Marx an Engels, 12. Dezember 1860 und 2. Februar 1861, MEW, Bd 30, S. 126, 152.

52 Ebd., S. 170.

53 J. F. Watson, zitiert nach Journal of the Society of Arts, and of the Institutions in Union (London), 19. April 1861; The Bengal Hurkaru. Overland Summary of News (Calcutta), 22. Juli 1861; Bombay Chamber of Commerce. Report for 1859-1860.

54 MEGA, Bd 3, T. 1, Berlin 1976, S. 315f.

55 MEW, Bd 30, S. 223f.; MEGA, Bd 3, T. 1, S. 294.

${ }_{56}$ MEGA, Bd 3, T. 2, Berlin 1977, S. 363ff.; MEW, Bd 26, T. 1, S. 40ff. Dort auch Ausführungen der Andeutungen zu Malthus, Lauderdale, Hodgskin, Say und Storch im Smithkommentar im Heft VII.

57 S. Anm. 173. 
Zunächst geht er den Ausführungen Smiths im fünften Kapitel des ersten Buchs über den ursprünglichen Zustand der Gesellschaft nach, in dem die Arbeit sowohl das natürliche $\mathrm{Ma} \beta$ des Tauschwerts sei als auch das Eigentumsverhältnis zwischen dem Arbeiter und dem Produkt seiner Arbeit herstelle. Dieses Eigentumsrecht werde dann infolge der Scheidung der Gesellschaft in die Klassen der Eigentümer und Nichteigentümer an den Produktionsmitteln und -bedingungen beschnitten, und zwar durch den Profit des Unternehmers und die Rente des Grundeigentümers. Smiths Bemerkungen zum Profit geben Marx ab Heftseite 186 Anlaß zu Abschweifungen über die Unterschiede zwischen Mehrwert und Profit, zwischen ihren Raten und zwischen Wert und Preis sowie prinzipiell zur Ableitung solcher Begriffe der Kritik der politischen Ökonomie. Zwei Seiten weiter kommt Marx wieder ,zurück zum Anfang”, d.h. zur Entstehung des Profits aus der Klassendifferenzierung der Gesellschaft, wonach der Arbeiter sein Produkt mit Kapitalisten und Grundeigentümern teilen müsse und weshalb die auf Beschaffung oder Produktion einer Ware verwandte Arbeit nicht mehr das einzige sei, was die Quantität Arbeit bestimme, welche diese Ware (als Subjekt) tausche, kaufe oder „kommandiere". Dasselbe Thema nimmt Marx dann in Smiths achtem Kapitel des ersten Buchs auf, worin dieser seine Vorstellung von der Entstehung des Profits und der Rente wiederholt und nochmals erläutert. Danach sucht Marx aus dem zweiten Buch Äußerungen Smiths über den Unterschied zwischen produktiver und unproduktiver Arbeit heraus und kehrt anschließend wieder zur Ausgangsproblematik zurück. Mit dieser beschäftigt sich auch der Anfang der folgenden englischen Exzerpte aus der Smithedition MacCullochs, die sich dann dem Verhältnis zwischen produktiver und unproduktiver Arbeit zuwenden. Hier hält Marx sich zunehmend kommentarlos an seine Vorlage und nimmt ab Heftseite 213 zusätzlich Zitate aus Arbeiten MacCullochs und William Pettys in seine Auszüge auf. Das auf Heftseite 218 folgende Exzerpt aus Ricardos Principles beginnt mit der bekannten Smithkritik des ersten Abschnitts über den Wert im ersten Kapitel des Buchs. Im Unterschied zum Ricardoexzerpt von 1851 nimmt Marx diesmal den vollen Wortlaut Ricardos kommentarlos auf.

Dem Smithkommentar liegen die alten Smithexzerpte von 1844, selbst wenn sie Marx zu einer ungefähren Orientierung gedient haben können, nicht zugrunde, da der Kommentar sachlich über sie hinausgeht. Trotz aller Abschweifungen kommt Marx immer wieder zur Fragestellung seines Anfangs zurück, nämlich zu Smiths Darstellung des Arbeitswerts und der Entstehung von Lohn, Profit und Rente. Dieser Gegenstand beherrscht den mit Abstand größten Teil des Kommentars. 
Der Abdruck folgt der Handschrift Marxens ohne Modernisierung oder Vereinheitlichung der Orthographie und der Interpunktion. Eindeutige Schreibverkürzungen im Wort werden stillschweigend ausgeschrieben, während alle mit einem Punkt versehenen Abkürzungen beibehalten sind. Marx schreibt französische und englische Texte sowie lateinische Worte in lateinischer, seinen eigenen Kommentar hingegen in deutscher Handschrift. Von Marx durch Unterstreichung hervorgehobene Buchstaben und Worte werden kursiv wiedergegeben, Randanstreichungen Marxens entsprechend reproduziert. Er schreibt durchgehend mit Tinte.

Zeichen:
$<>$ Tilgung
$|:|$ Einfügung
$\mathrm{x} \quad$ unlesbarer Buchstabe
$>\quad$ Veränderung zu
xxx unlesbare Buchstabengruppe
// Beginn einer Heftseite

1. I, ch. $6^{\mathrm{b}}$. La division ${ }^{58}$ une fois établie $\mathrm{e}^{59}$ - il (l'homme) ${ }^{60}$ sera riche ou pauvre, selon la quantité du travail qu'il pourra commander ou qu'il sera en état d'acheter. ${ }^{61}$ Ainsi la valeur d'une denrée quelconque pour celui qui la possède, et qui n'entend pas en user ou la consommer lui-même, mais qui a intention de l'échanger pour autre chose, est égale à la quantité ${ }^{62}$ de travail que cette denrée le met en état d'acheter ou de commander. (t. I, 59, 60. Edit. Garnier. 1802) Comme dit Mons. ${ }^{63}$ Hobbes, richesse veut dire pouvoir .... droit de commandement sur tout le travail d'autrui ${ }^{64}$. (p. 61 . l.c.) Sa fortune est plus ou moins grande exactement, en proportion de l'étendue de ce pouvoir, en proportion c de la quantité du travail d'autrui,

a $<1.1 .>$

$\mathrm{b}$ unsicher; möglicherweise verändert aus richtig 5

$c<$ de l'étendue de ce pouvoir $>$

${ }^{58}$ Bei Adam Smith, Recherches sur la nature et les causes de la richesse des nations. Traduction nouvelle, avec des notes et observations; par Germain Garnier, de l'Institut National. Avec le portrait de Smith, 5 Bde, Paris 1802, Bd 1, Kap. 5, S. 59: Mais la division

${ }^{59}$ Bei Smith, S. 59: établie dans toutes les branches du travail, il n'y a qu'une partie extrêmement petite de toutes ces choses qu'un homme puisse se procurer directement par son travail, et c'est du travail d'autrui qu'il lui faut attendre la très-majeure partie de toutes ces jouissances; ainsi il sera

${ }^{60}$ Einfügung Marxens.

61 Bei Smith Absatz.

${ }^{62}$ Bei Smith Übergang zu S. 60.

63 Bei Smith: M.

${ }^{64}$ Bei Smith: d'autrui, ou sur tout le produit de ce travail existant alors au marché 
qu'elle le met en état de commander, ou ce qui est la même chose, du produit du travail d'autrui, qu'elle le met en état d'acheter. (61) Il est . .65 plus naturel d'estimer sa valeur échangeable ${ }^{66}$ [d'une chose] par la quantité qu'elle peut acheter de quelque autre ${ }^{67}$ denrée que par celle du travail qu'elle acheterait. $\left(63^{68}\right)$

Dans ce premier état informe de la société, qui précède l'accumulation des capitaux et la propriété des terres, la seule circonstance qui puisse fournir quelque règle pour les échanges, c'est, à ce qu'il semble, la quantité de travail nécessaire pour acquérir les différens objets d'échange ${ }^{69}$. . . Il est naturel que ce qui est ordinairement le produit de deux jours ou de deux heures de travail, vaille le double de ce qui est ordinairement le produit d'un jour ou d'une heure de travail. $(94,95)$ In diesem Zustand der Werth bestimmt durch d. Quantität der in d. d Waare enthaltnen Arbeit; aber auch d. Arbeiter sein ganzes Product gehörig, od. in andren Worten: die Waare commandirt (kauft) nur so viel Arbeit als in ihr enthalten ist. Es ist wenigstens wichtig, daß A. Smith e ausdrücklich festsetzt, daß f d. Gesetz d. Tauschwerths nach, wenn kein spezifischer Unterschied zwischen Arbeit $u$. andern Waaren, kein Profit möglich wäre. Er sagt daher ausdrücklich:

„Dans cet état de choses, le produit du travail appartient tout entier à l'ouvrier, et la quantité de travail communément employée à acquérir ou à produire un objet échangeable est la seule circonstance qui puisse régler la quantité de travail que cet objet devrait communément acheter, commander ou obtenir en échange." $(96)^{70}$

Nun d. Uebergang:

„Aussitôt qu'il y aura des provisions accumulées dans les mains de quelques particuliers, certains d'entr'eux emploieront naturellement ces provisions à mettre en cuvre des gens industrieux, auxquels ils fourniront des matériaux et des subsistances, afin de faire un profit sur la vente de l'ouvrage, ou sur ce que le travail de ces ouvriers ajoute de valeur aux matériaux. ${ }^{71}$ [Aber wo kommen d. gens industrieux her, die weder Subsistenzmittel noch Material der Arbeit besitzen, in die blaue Luft gestellt sind? Entkleidet man Smith's Ausdruck seiner naiven Fassung, so heißt er weiter

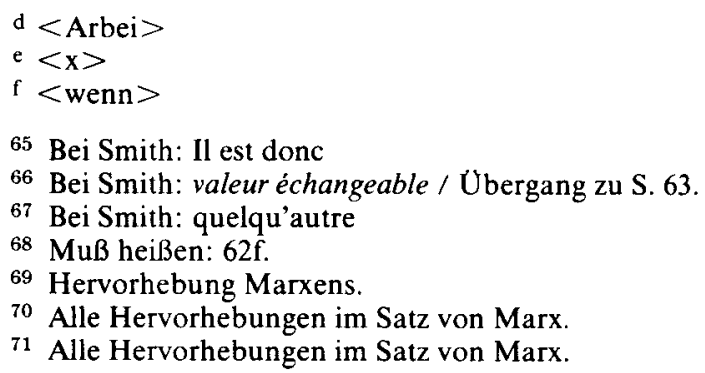


nichts als: die capitalistische Production beginnt in dem Augenblick, wo die Arbeitsbedingungen einer Klasse angehören u. die blose Verfügung über ihr Arbeitsvermögen einer andern. Diese Trennung in d. Bedingungen d. Arbeit bildet d. Voraussetzung d. capit. Production. Zugleich aber erklärt Smith, daß d. Profit sur la vente de l'ouvrage nichts andres ist als der profit sur ce que le travail de ces ouvriers ajoute de valeur (also selbst g quantité de travail) aux matériaux.” (p. 96) Er fährt fort: „Quand l'ouvrage fini est échangé, ou contre de l'argent, ou contre du travail, ou contre d'autres marchandises, il faut bien qu'en outre de ce qui pourrait suffire à payer le prix des matériaux et les salaires des ouvriers, il y a $\mathrm{a}^{72}$ encore quelque chose de donné pour les profits de l'entrepreneur de l'ouvrage, qui hasarde ses provisions dans cette affaire ${ }^{73}$ (1c) [Hier also d. Grund d. Profits auf d. hasarder des provisions, d. Risico, das d. Waaren durch ihr Eingehn in d. Productionsprozeß laufen, zurückgeführt. Dieß richtig in d. gegenseitigen Bereichrung d. Capitalisten unter sich. Sinnlos d. Arbeitern gegenüber. Es bliebe nichts übrig als d. Provisions aufzuessen, mit retainers, wie es d. feudalen thaten; od. sie in d. Form v. Schatz, Diamanten, if il y en a brach liegen, od. sie verfaulen zu lassen. D. Risico d. Production ist kleiner als d. andre. Wo es daher an Arbeitskräften fehlt, Rußland, h Buenos Ayres etc ein Theil d. Provisions [Siehe Storch] nutzlos weggeworfen ${ }^{i}$ etc]. Dieß Risico also Blödsinn. Ferner noch zu bemerken in dieser Stelle: Derj fact, daß d. Capitalist einen Theil d. Arbeit, den d. Arbeiter dem Material zufügt, sich aneignet, kann doppelt aus gedrückt werden $u$. wird doppelt ausgedrückt v. A. Smith: Erstens: Verkauft er d. Waare zu ihrem Werth, der in ihr enthaltnen Arbeitszeit, d.h. tauscht er sie gegen andre Waaren nach d. Gesetz d. Werths aus, so rührt sein Profit daher, daß er einen Theil der in d. Waare enthaltnen Arbeit nicht bezahlt hat. Zweitens: Tauscht er $\mathrm{d}$. Waare wieder gegen Arbeit aus, so kauft er mehr lebendige Arbeit damit, als vergangne in ihr enthalten ist. Denn schon in casus I ist unterstellt, daß er nicht gleiches Quantum vergangner Arbeit gegen gleiches Quantum lebendiger Austauscht; daß d.v. ihm angeeignete Quantum lebendiger Arbeit k kleiner ist als d. Quantität v. ihm bezahlter lebendiger Arbeit. Im letztern Fall würde d. Lohn d. Arbeiters /174/ gleich s. Product sein. Casus 2) ist sogar d. Voraussetzung v. casus I. Nur Wie-

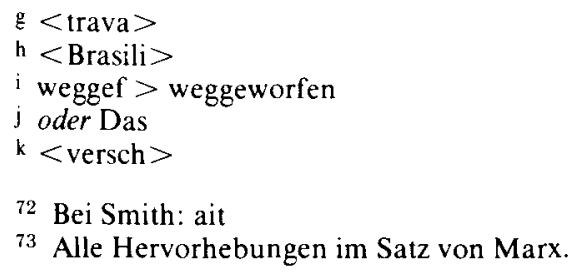


derholung derselben Operation. Dieß hat d. Anstoß zur Malthus'schen Theorie d.Werthes gegeben] ,Ainsi la valeur que les ouvriers ajoutent à la matière se résout alors en deux parties, dont l'une paie leurs salaires et l'autre paie les profits que fait l'entrepreneur ${ }^{74}$ sur la somme des fonds qui lui ont servi à avancer ces salaires et la matière à travaillera. ${ }^{75}$ (p. 96 ${ }^{76}$ ) Also d. Profit ist nichts als ein Abzug v. d. Werthb, den d. Arbeiter dem Material hinzugefügt haben. Nun besteht $d$. Werth aber nur in d. Quantität Arbeit. Die Sache löst sich also, wenn A. Smith selbst seinen Prämissen entsprechend sich ausgedrückt, dahin aufc: daß d d. Arbeitszeit d. Arbeiters sich in zwei Theile auflöst, der eine, für den er ein Equivalent vom Capitalisten erhält, s. Salair, der andre, den er ihm gratis giebt u. der den Profit constituirt. Unterscheidung v. Mehrwerth u. Profit befindet sich noch nicht bei Adam, obgleich er richtig hervorhebt, daß nur d. Theil Arbeit (Werth), den der Arbeiter dem Material hinzufügt, sich in Salair u. Profit auflöst; also d. e Mehrwerth an u. für sich nichts zu thun hat mit dem ausgelegten (als Material u. Instrument) Theil d. Capitals. f A. Smith glaubt daß d. Werthbestimmung durch Arbeitszeit aufhört, weil sie on first view dem Austausch zwischen Capital u. lebendiger Arbeit widerspricht. Ricardo zeigt daß das nicht $d$. Fall ist $u$. daß d. Waaren sich trotz Capital $u$. Grundeigenthum nach d. Quantität in ihnen enthaltner Arbeitszeit austauschen. Er zeigt nicht noch, untersucht nicht, warum d. beiden Ausdrücke: quantity of labour worked up in a commodity and quantity of labour which a commodity may command nicht identisch sind. Nimmts als fact. Malthus dreht d. Sache um. Der Werth d. Waare bei ihm nur bestimmt durch ihren Werth als Capital, d.h. d. Arbeit die sie mehr kaufen kann als sie selbst enthält.] A. Smith fährt fort:

„Il (l'entrepreneur) n'aurait pas d'intérêt à employer ces ouvriers, s'il n'attendait pas de la vente de leur ouvrage quelque chose de plus que ce qu'il fallait pour lui remplacer ses fonds, et il n'aurait pas d'intérêt à employer une grosse somme de fonds plutôt qu'une petite, si ses profits ne gardaient pas quelque proportion avec l'étendue des fonds employés."77 (97)

A. Smith zeigt dann noch daß d. ,profit des fonds" (97, sq.) durchaus

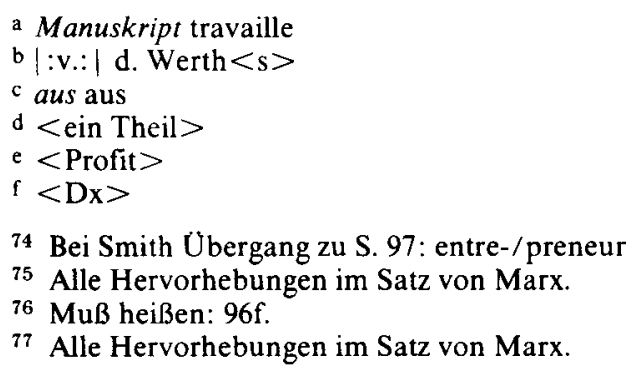


nicht un nom différent ist donné aux salaires $g$ d'une espèce particulière de travail, le travail d'inspection et de direction. Ils sont $\mathrm{h} 78$ d'une nature absolument différente des salaires; ils se règlent sur des principes entiérement différens, et ne gardent aucune proportion ${ }^{79}$ la quantité et la nature de ce prétendu travail d'inspection et de direction. Ils se règlent en entier sur la valeur du capital employé, et ils sont plus ou moins forts, à proportion de l'étendue des fonds. ${ }^{80}$ (97) D. Beispiel was Smith nun (97 u. 98) bringt, beruht darauf: in 2 i entreprises 20 ouvriers angewandt, 151 . per an, $=3001$. Das Material d. einen koste 700 1., das d. andern 7000, d. Capital angewandt in dem einen $=1000 \mathrm{l}$, in $\mathrm{d}$. andern $=7300$. Das eine zu $10 \%$ wird 100 l. Profit machen, d. andre 730. Dennoch kann ihre Arbeit d'inspection et de direction dieselbe sein. [Dieß Beispiel gut für das was Smith beweisen will. Schlimm f. s. Entstehung d. Profits. Denn wenn nicht angenommen wird, daß d. Arbeiter in einem höhern Material, was wohl meistens d. Fall, höhere Arbeit liefern, wie diesen höheren Profit erklären, da 20 Arbeiter d. Material, was es auch immer koste, nur 20 Arbeitstage zusetzen können. Offenbar $d$. höherek Profit hier nur entspringend ${ }^{l}$ aus $d$. ausgleichenden Vertheilung des Gesammtprofits (surplus value), den d. Capitalistenklasse macht. Diese im Preiß d. Products vermittelt. D. Verwechslung $v$. surplus value $u$. profit macht hier jede wissenschaftliche Betrachtung unmöglich. Wenn d. eine 7000 auslegt, der andre 1000, u. der m. 7000 nur 100 Profit machte, so würde er also 700 machen, wenn er s. ganzes Capital in d. andern Geschäftszweig unterbrächte. Die Concurrenz würde d. Zufuhr im $2^{t}$ Zweig so klein machen, daß $m$ er Monopolprofite machen würde, sie in d. andern herabdrücken etc] Ferner sieh was A. Smith über d. commis sagt, d. in fact d. ganze travail de direction leitet. (p. 98, 99) Also Smith gegen d. wages of labour of superintendence. Nach dieser Entwicklung fährt er fort:

„Ainsi, dans le prix des marchandises, les profits des fonds ou capitaux sont une source de valeur entiérement différente des salaires, et réglée sur des principes tout-à-fait différens."81 (99.) So weit dieß gegen d. labour of

$\mathrm{g}<$ du travail $><$ d $>$

$\mathrm{h}$ unentzifferbares Zeichen

i $\langle\mathrm{xxx}>$

j 7 verbessert aus $\mathrm{x}$

${ }^{k}$ nachträglich eingefügt

1 unsicher; Handschrift entsprgden

$\mathrm{m}<$ sie $>$

${ }^{78}$ Bei Smith: Les profits des fonds, dira-t-on peut-être, ne sont autre chose qu'un nom différent donné [...]. Ils sont cependant

79 Bei Smith: proportion avec

${ }^{80}$ Alle Hervorhebungen im Zitat von Marx.

81 Alle Hervorhebungen im Satz von Marx. 
superintendence gerichtet, richtig. Sonst ganz falsch. Nach Smith eben noch vertheilt sich d. valeur, den d. n Arbeiter d. Material zufügen, zwischen ihnen u. Capitalisten, zwischen salaires u. profits; ist also d. einzigeo source de valeur, $u$. aus dieser source de valeur entspringen prix de salaires u. prix de profits. Aber diese prix selbst sind keine source de valeur. Weder d. Arbeitslohn noch d. Profit. Also falsch v. d. profits als source de valeurs zu sprechen. [In diesem Punkt Lauderdales Polemik gegen Smith richtig.] Versteht Smith, wie er hier thut, unter prix nichts als d. monetary expression of the relative value of commodities, so $s$. Satz ganz falsch. Versteht er Marktpreiß u.s.w. darunter, so relativ richtig. Aber d. Gesetze d. prix in diesem Sinne können überhaupt nur entwickelt werden, nachdem die Gesetze d. Werths u. d. ursprünglichen Verhältnisses v. salaires $u$. profits dazu entwickelt sind. $p$

/186/a D. Profit (d. Grund desselben) reducirt A. Smith wieder, 1. I, ch. IX (t. I p. 196) auf d. Risico in d. Production. ${ }^{82}$ [u. dieß ist richtig, nicht so fern es d. Profit d. Capitalisten betrifft; d. Aneignung eines Theils d. surpluslabour durch eine besondre Klasse, sondern so weit selbst unter $d$. Voraussetzung, daß d. Arbeiter Herren ihres Products, sie immer mehr arbeiten müßten, um Reservefonds zu haben, über ihre zur Subsistenz nöthige Arbeitszeit hinaus.)

„le taux le plus bas des profits ordinaires des capitaux doit toujours être quelque chose au dela ${ }^{83}$ de ce qu'il faut pour compenser les pertes accidentelles auxquelles est exposéb chaque emploi de capital. Il n'y a que ce surplus qui constitue vraiment le profit ou le bénéfice net." ${ }^{84}$ (p. 196) (Constituirt aber nur so weit als es Vorwand ist, d. Verlust nicht wirklich gemacht wird. Profit of insurance) (Smith sagt auch hier: es müsse mehr sein als d. Risico ,ce qu'on retient pour la compensation de ces pertes extraordinaires."

Wie wenig d. Profit m. d. salaire gemein hat, setzt A. Smith weiter aus einander c 1. I, ch. X (t. I, p. 205 sqq.) D. salaires de travail wechseln nach 5 Umständen: 1) l'agrément ou le desagrément des emplois eux mêmes ${ }^{85}$.

$\mathrm{n}<\mathrm{C}>$

0 nachträglich eingefügt

p am Ende der Seite (Fts. p. 186)

a am Anfang der Seite (Ftsg v. p. 174. A. Smith) (1. I, ch. VI

$\mathrm{b}$ aus exposée

c $<$ 1. II, c. 5. $>$

82 Bei Smith, S. 196: Ce qu'on nomme gros profit comprend souvent, non seulement ce surplus, mais encore ce qu'on retient pour la compensation de ces pertes extraordinaires.

${ }^{83}$ Bei Smith: Le taux $[\ldots]$ au-delà

${ }^{84}$ Alle Hervorhebungen im Zitat von Marx.

${ }^{85}$ Bei Smith, S. 203: désagrément des emplois en eux-mêmes 
Dieß trifft in gewissem Maaß auch bei den Capitalien zu. 2) la facilité et le bon marché avec lequel on peut les (emplois) apprendre, ou la difficulté et la dépense qu'ils exigent pour cela. Dieß trifft nicht bei d. Capitalien zu. Quant aux profits de capitaux, ils semblent être très peu ${ }^{86}$ affectés par la facilité ou la difficulté de se mettre au fait du genre d'affaires auquel on les emploie." 3) ,l'occupation constante qu'ils procurent, ou les interruptions auxquelles ils sont exposés. Dieß trifft wieder bei d. Capital nicht ein. „les profits ordinaires de ${ }^{87}$ capitaux ne peuvent, dans aucun commerce particulier, être affectés de la constance ou de l'interruption de l'emploi. C'est la faute du capitaliste, et non celle des affaires, si le capital n'est pas constamment employé." 4.) le plus ou moins de confiance dont il faut que soient investis ceux qui les exercent." Dieß trifft wieder nicht ein beim Capital. „Quand une personne n'emploie au commerce d'autres capitaux que les siens propres, il n'y a pas lieu à confiance, et le crédit qu'elle peut d'ailleurs se faire dans le public, ne dépend pas de la nature de son commerce, mais de l'opinion qu'on a de sa fortune, de sa probité et de sa prudence. Ainsi les diverses branches de commerce $^{88}$ ne peuvent pas provenir des différens degrés de confiance accordés aux capitalistes qui le font." 5) la probabilité ou l'improbabilité d'y réussir." Dieß einigermaassen beim Capital. „Dans tous les divers emplois de capitaux, le taux ordinaire du profit varie plus ou moins, suivant le plus ou moins de certitude des rentrées. ${ }^{89} \ldots$ Le taux ordinaire de ${ }^{90}$ profit s'élève toujours plus ou moins avec le risque. Il ne paraît pas pourtant qu'il s'élève à proportion du risque, ou de manière à le compenser parfaitement."

Die Behauptung d. économistes, daß d. profit nichts ist als wages for $d$ labour of superintendence, hat ihre Gegner dahin geführt zu verlangen, $\mathrm{da} \mathrm{d}$. profits nur hierauf - auf diese wages of superintendence - reducirt werden sollen. (Hodgskin. Cooperative manufacture. .)

Die falsche Ansicht Smiths (mit s. eignen richtigen Entwicklung im Widerspruch) v. d. prix de salaires etc als constituant la valeur, führt weiter:

„Dans toute société, le prix de chaque marchandise se résout définitivement en quelqu'une ${ }^{91}$ de ces trois parties (profit, salaire ${ }^{92}$, rente) ou en

d $<$ the $>$

${ }^{86}$ Bei Smith, S. 209: très-peu

87 Bei Smith, S. 213: des

${ }^{88}$ Bei Smith, S. 215: les différens taux du profit dans les diverses branches du commerce

${ }^{89}$ Smith, S. 226.

${ }^{90}$ Smith, S. 227: du

91 Bei Smith Übergang zu S. 101.

${ }^{92}$ Smith spricht hier von ,travail”. 
toutes trois. . Ces trois parties semblent constituer immédiatement ou en définitif la totalitép ${ }^{93} \mathrm{du}$ prix du blé (l.c.e) Abgesehn davon daß d. prix so den prix constituirt etc, falsch d. totalité. Smith fährt zwar fort: „On pourrait peut-être penserf qu'il faut y ajouter une quatrième partie, nécessaire pour remplacer le capital du fermier ou pour compenser le dépérissement et l'user de ses chevaux de labour et autres instrumens d'agriculture. Mais il faut considérer que le prix de tout instrument de labourage, tel qu'un cheval de charrue, est lui-même formé de ces mêmes trois parties." (1. I ch. VI) (t. I, p. 101 ${ }^{94}$ ) Il faut plutôt considérer, daß auch d. Pferdezüchter / $187 /$ ein Vorschußcapital nöthig hat $u$. daß d. fonds, a der ihm salaires, profits, rentes zahlt, nichts andres ist, als $\mathrm{d}$. Mehrwerth, den d. Arbeit diesem vorhandnen Werthbetrag zugesetzt hat. Wie ein Theil d. Samens u.s.w. der Erde zurückgegeben werden muß, u. weder inb Profit, Salair, noch Grundrente aufgehn kann, so in jeder $c$ industriellen entreprise Rohstoff u. Arbeitsinstrument. Dieß f. d. Gesellschaft, wie f. d. einzelnen. Er ist ein Theil d. vorhandnen Products, der d beständig reproducirt $u$. beständig abgezogen wird. Er bildet d. eigentlichen Productionsfonds d. Gesellschaft. Und jede Waare, so weit sie nicht rein nur durch Aneignung d. vorhandnen Naturproducts entsteht, enthält in sich einen solchen Theil (d.h. in ihrem Werth), der diesen fonds ersetzt u. eine revenue wird. Es ist faktisch dasselbe als wenn jeder einen Theil s. Products nicht verkaufte, sondern d. aufgezehrten fonds daraus ersetzt. Jedes Jahr muß ein Theil d. Products als Rohstoff u. Arbeitsinstrument in d. Production eingehn u. kann weder fonds für salaires, profits, noch rentes bilden. Er besteht auch aus Arbeit, aber Theil d. Arbeit, der keine Revenue bildet. A. Smith geräth noch in grosse Verlegenheit, e sobald er seine eigne Theorie auf ein ganzes Land anwenden soll.

„On a fait voir ... que le prix de la plupart des marchandises se résout en trois parties, dont l'une paie les salaires du travail ${ }^{95}$, l'autres les profits du capital $^{96}$, et la troisième la rente de la terre ${ }^{97}$. (1. II, ch. II) (t. II, p. $213 .{ }^{98}$ )

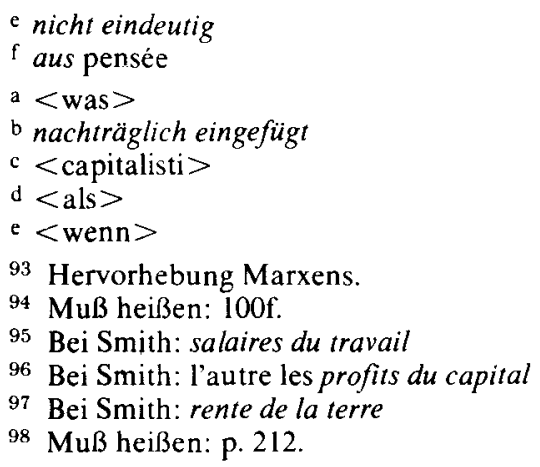


Also löst sich d. ganze Preiß einer Waare in Revenue auf. $f$ Also könnte auch ein Land d. jährliche Product seiner Arbeit, d. revenu brut, in Form dieser 3 Revenuen jährlich verzehren. Aber quod non, sagt Adam - u. necessarily so. „On a observé que puisqu'il en était ainsi pour toute marchandise quelconque prise séparément, il fallait nécessairement qu'il en fût de même pour les marchandises qui composent la totalité ${ }^{99}$ du produit de la terre et du travail d'un pays, prises en masse. La somme totale du prix ou de la valeur échangeable de ce produit annuel doit se résoudre de même en ces trois parties et se distribuer entre les différens habitans du pays, ou comme salaires ${ }^{100}$ de leur travail, ou comme profits ${ }^{101}$ de leur capital, ou comme rentes ${ }^{102}$ de leur terre. (l.c. 1. II, ch. II) (p. 213, t. II) Aber wie hilft sich Adam nun heraus:

„Mais quoique la valeur totale du produit annuel des terres et du travail d'un pays soit ainsi partagée entre les différens habitans et leur constitue un revenug, cependant, de même que dans le revenu d'un domaine particulier, nous distinguons le revenu brut et le revenu net ${ }^{103}$, nous pouvons aussi faire une pareille distinction à l'égard du revenu de tous les habitans d'un grand pays. ${ }^{104}$ Le revenu brut d'un domaine particulier comprend ${ }^{105}$ généralement tout ce que débourse le fermier; le revenu net est ce qui reste franc et quitte de toutes charges au propriétaire, après la déduction des frais de régie etc ${ }^{106} \mathrm{Sa}$ richesse réelle n'est pas en proportion de son revenu brut, mais bien de son revenu net. ${ }^{107}$ Le revenu brut de tous les habitansh d'un grand pays comprend la masse totale du produit annuel de leur terre et de leur travail; leur revenu net est ce qui leur reste franc et quitte, déduction faite de ce qu'il faut pour entretenir premièrement leur capital fixe: ${ }^{108}$ secondement, leur capital circulant; ou bien ce qu'ils peuvent placer, sans empiéter sur leur capital, dans leur fonds de consommation, c.à.d. ${ }^{109}$ qu'ils peuvent dépenser pour leur subsistance, commodités et amusemensi. Leur

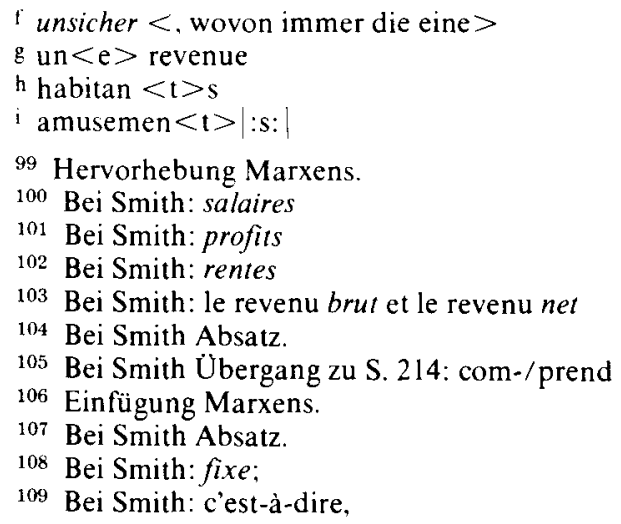


richesse réelle est aussi en proportion de leur revenu net et non pas de leur revenu brut." (p. 213j 214) (t. II) (1. II, ch. II) Also d. revenu brut eines particulier wie einerk Gesellschaft löst sich nicht auf in salaires, profits $u$. rentes, $d$. in d. fonds de consommation gehn können, sondern eines Theils d. Capitals d. als Werth (also als Gebrauchswerth) beständig für d. Reproduction selbst ersetzt werden muß u. nie Revenue werden kann. Also besteht auch d. prix d. Waaren aus d. Prix dieses d. Production selbst ${ }^{1}$ angehörigen $u$. beständig erneuerten Theils d. Production. Say auf Smithm gestützt stellte mehr auf, daß d. ganze $\mathrm{n}$ revenu brut revenu net sei. Dagegen Storch.

„Il est évident", sagt Smith ,qu'il faut retrancher du revenu net de la société, toute la dépense d'entretien du capital fixe. Ni les matières nécessaires pour la conservation des machines utiles, des instrumens de métier, bâtimens d'exploitation, etc. ni le produit du travail nécessaire pour façonner ces matières dans la forme convenable, ne peuvent jamais faire partie de ce revenu net. Le prix ${ }^{110}$ de ce travail [hier bringt er ganz ungehöriges hinein), à la vérité, peut bien en faire partie, puisque les ouvriers qui y sont employés, peuvent placer la valeur entière de leurs salaires dans leur fonds de consommation. Mais la différence, c'est que, dans les autres sortes de travail, et le prix et le produit vont / 188/l'un et l'autre à ce fonds; le prix va à celui des ouvriers, et le produit à celui d'autres personnes, ${ }^{111}$ dont la subsistance, les aisances et les amusemens se trouvent augmentés par le travail de ces ouvriers. (t. II, p. 214, 15) (b. II, ch. II) „La destination du capital fixe est d'accroître la puissance productive $\mathrm{de}^{112}$ travail, ou de mettre le même nombre d'ouvriers à portée de faire une beaucoup plus grande quantité d'ouvrage”. (p. 215 l.c.) p. 220 sagt er sogar: ,de même que les machines et instrumens de métier etc, ${ }^{113}$ qui composent le capital fixe ${ }^{114}$, soit d'un individu, soit d'une société, ne font partie ni du revenu brut ${ }^{115} \mathrm{ni} \mathrm{de}^{116}$ revenu net de l'un ou de l'autre, de même l'argent ... ne fait

\footnotetext{
$\mathrm{j}$ nachträglich eingefügt

$\mathrm{k}$ eine $<\mathrm{s}>\mathrm{r}<$ Staats $>$

1 nachträglich eingefügt

$\mathrm{m}<$ Ricardo $>\mid:$ Smith: $\mid$

$\mathrm{n}<$ bru $>$

110 Hervorhebung Marxens.

111 Komma von Marx.

112 Bei Smith: du

113 Bei Smith: métier, etc.

114 Bei Smith: capital fixe

115 Hervorhebung Marxens.

116 Bei Smith: du
} 
nullement partie ${ }^{117}$ de ce revenu. (Das durch es vertheilt ist.) Dagegen meint er:

„Mais quoique toute la dépense d'entretien du capital fixe ${ }^{118}$ se trouve ainsi nécessairement retranchée du revenu net de la société, il n'en est pas de même à l'égard de la dépense d'entretien du capital circulant... Des quatres $^{119}$ articles qui composent ce capital, qui sont l'argent, les vivres, les matières et l'ouvrage fait les trois derniers en sont réguliérement retirés pour être versés, soit dans le capital fixe ${ }^{120}$ de la société, soit dans le fonds de consommation ${ }^{121}$. De ces choses consommables, tout ce qui ne se trouve pas employé à l'entretien du premier de ces deux fonds, va en entier à l'autre, et fait partie du revenu net de la société: ainsi l'entretien de ces 3 parties du capital circulant ${ }^{122}$ ne retranche du revenu net de la société aucune autre portion du produit annuel, que celle qui est nécessaire à l'entretien du capital fixe. ${ }^{123}$ À cet égard, le capital circulant d'une société diffère de celui d'un individu." (t. II. 218) (1. II. ch. 2)

D'abord vivres vom Vieh gefressen. Ein Theil d. matière ist jedes Jahr a Arbeitsmaterial, kann also ebenso wenig in d. revenu eingehn ${ }^{b}$. Kommen wir zurück zum Anfang.

„Dans cet état de choses donc, (wo d. Capitalist vom Arbeiter unterschiedene Person ist) le produit du travail n'appartient pas toujours tout entier à l'ouvrier. Il faut le plus souvent que celui-ci partage avec le propriétaire $d u^{124}$ capital qui le fait travailler. Ce n'est plus alors la quantité de travail mise communément à acquérir ou à produire quelque marchandise, qui est la seule circonstance qui puisse régler la quantité de travail que cette marchandise devra communément acheter, commander ou obtenir en échange. ${ }^{125}$ Il est clair qu'il sera encore dû une quantité additionnelle pour le profit du capital qui a avancé les salaires de ce travail et qui en a fourni les matériaux. (1. I, ch. VI) (t. I, p. 99) (Er fährtc naiv fort: De l'instant que le sol d'un pays est divisé en autant de propriété privées, les propriétaires, comme tous les autres hommes, aiment à recueillir où ils n'ont pas semé, et

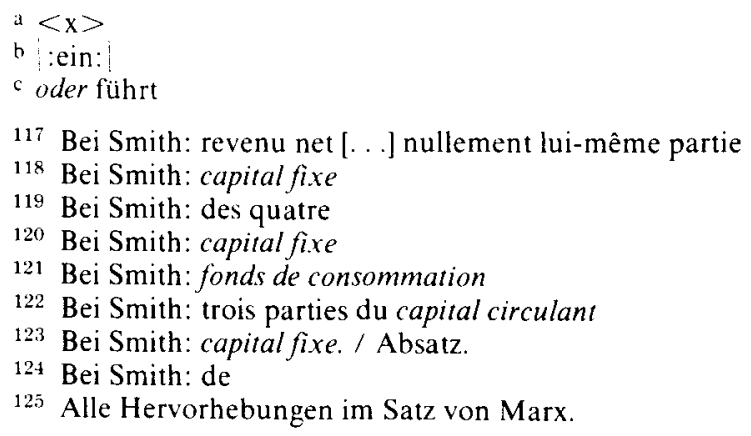


ils demandent une rente, même pour le produit naturel du pays. ${ }^{126 "}$ " l.c.)

Also: weil die in d. Waare enthaltene Arbeit nicht mehr $=\mathrm{d}$. Arbeit, die mit d. Waare gekauft werden kann (d. Capitalist kauft vielmehr mehr Arbeit $m$. d. Waare, als in ihr enthalten ist $u$. die $\beta$ ist d. fonds d. profit), hört die in d. Waare enthaltne Arbeit auf Maaß d. Werths für A. Smith zu sein. Dagegen Ricardo. Oder Smith glaubt daß dieß Werthmaaß aufgehört hat, weil es prima facie nicht anwendbar ist auf $d$. Verhältniß v. Capital $u$. Lohnarbeit. Er d nimmt daher als Maaß d. Werthe (was er jedoch nirgend durchführen kann, weil es in sich absurd ist) d. Quantum Arbeit, das ich mit einer Masse Waaren kaufen kann, od. e d. Masse Waaren, die ein bestimmtes Quantum Arbeit erstehn kann, ein Quantum was ganz v. d. Grösse d. Salairs abhängt einerseits. Dieß hat Malthus nachher aufgenommen. So sagt Smith weiter: „Il faut observer que la valeur réelle de toutes les différentes parties constituantes du prix se mesure par la quantité de travail que chacune d'elles peut acheter ou commander. Le travail mesure la valeur, non-seulement de cette partie du prix qui se résout en travail $^{127}$, mais encore de celle qui se résout en rente, et de celle qui se résout en profit." (p. 100. t. I) (1. I. ch. VI)

A. Smith resümirt noch einmal s. ganze Ansicht, wobei es erst recht klar wird, wie wenig $f$ er irgend wie $g$ auch nur zu begründen sucht, daß $d$. Werth, den der Arbeiter d. Product zufügt, h (nach Abzug d. frais de production) i nicht mehr durch d. im Product enthaltne Arbeitszeit bestimmt sein soll, weil d. Arbeiter $\mathrm{j}$ sich diesen Werth nicht ganz aneignet, sondern ihn mit capitaliste $u$. propriétaire zu theilen hat, d. Werth od. d. Product. D. Art wie ein Werth vertheilt wird, ändert natürlich nichts an s. Natur.

1. I ch VIII „Ce qui constitue la récompense naturelle ou le salaire du travail, c'est le produit du travail. ${ }^{128}$ [récompense naturelle ist hier nach Weise d. $18^{t} \mathrm{Jhh}$. Naturzuständliche récompense]. Dans cet état /189/ primitif qui précède ${ }^{129}$ la propriété des terres et l'accumulation des capitaux, le produit entier du travail appartient à l'ouvrier. Il n'a ni propriétaire

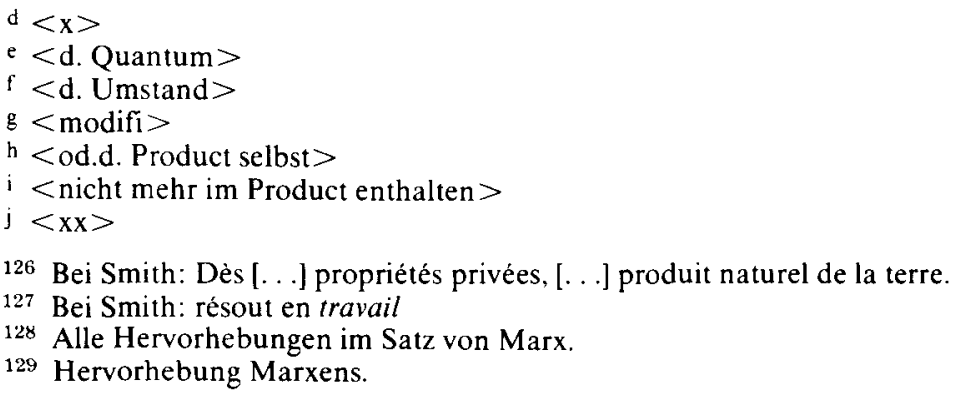


ni maître avec qui il doit ${ }^{130}$ partager. ${ }^{131}$ Si cet état eût continué, le salaire du travail aurait augmenté $\mathrm{a}^{132}$ mesure que ses facultés productives auraient acquis toutes ces améliorations ${ }^{133}$ auxquelles donne lieu la division du travail. [Also, par contrea, sagt A. Smith, daß mit Capital u. propriétéb d. salaire d. Arbeit nicht wächst $\mathrm{m}$. d. Entwicklung $\mathrm{c} d$. Productivkräfte ihrer Arbeit. Elles ${ }^{134}$ auraient été produites par de moindres quantités de travail, et elles auraient été pareillement achetées avec le produit de moindres quantités, puisque, dans cet état de choses ${ }^{135}$, des marchandises produites par des quantités égales de travail se seraient naturellement changées ${ }^{136}$ l'une contre l'autre." (1. I. ch. VIII. t. I. p. 129) Mais cet état primitif dans lequel l'ouvrier jouissait de tout le produit de son propre travail, ne peut pas durer audelà ${ }^{137}$ de l'époque où commencèrent à s'introduire la propriété des terres et l'accumulation des capitaux. Il y avait donc longtemps ${ }^{138}$ qu'il n'existait plus quand les facultés productives du travail ${ }^{139}$ ont acquis un degré de perfection considérable etc. ${ }^{140}$ (l.c. t. I, p. 131) Sa (d. propriétaire) rente est la première déduction ${ }^{141}$ que souffre le produit du travail appliqué à la terre. (l.c. p. 131.) Le produit de presque tout autre travail est sujet à la même déduction ${ }^{142}$ en faveur du profit. . . Ce maître [d. capitalist] partage dans le produit de leur travail ou dans la valeur que ce travail ajoute à la matière à laquelle il est appliqué, et c'est cette part qui constitue son profit." (p. 152) ${ }^{\mathbf{1 4 3}}$ l.c. A. Smith bezeichnet also ausdrücklich Rente u. Profit als blose Abzüge v. d. Product d. Arbeiters od. d. Werth dieses Products $=\mathrm{d}$. v. Arbeiter zugefügten Arbeit. Capital u. propriété ändern ihm also nur an d. Aneignung od. Vertheilung (ursprünglichen) d. Products od. seines Werths, d.h. d. in ihm enthaltnen Quantität Arbeit. Weder sind sie source de valeur, noch können sie etwas ändern an d. Natur

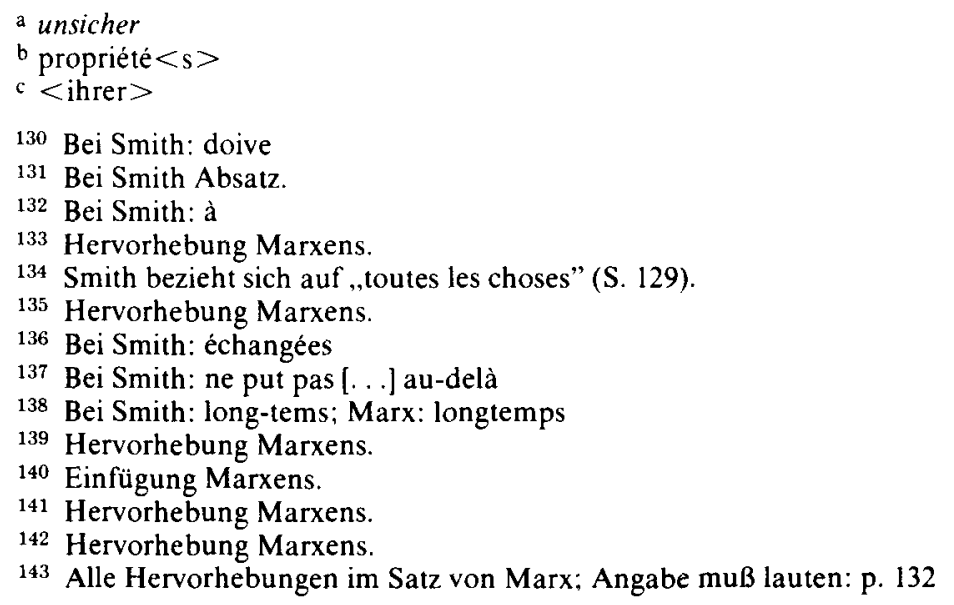


d. valeur. Er hätte also umgekehrt sagen müssen: Mit capital u. propriété, d da d. e Product d. Arbeit nicht mehr d. Lohn d. Arbeit, d. Quantität Waaren, womit man Arbeit kauft, od. d. Quantität $\mathrm{f}$ Arbeit, womit man Waaren kauft, nicht mehr $=$ sind der in d. Waaren enthaltnen Quantität Arbeit, hört d. Belohnung d. Arbeit od. d. Quantität Arbeit, die man mit $g$ einer bestimmten Waare kaufen kann, auf Werthmaaß zu sein. Sie war es nur so lang, als sie dem in d. Waare selbst enthaltnen Quantität Arbeit gleich war. Er schließt umgekehrt.

Es giebt, sagt A. Smith, ouvriers, d. m. ihren eignen Instrumenten arbeiten $u$. sich selbst d. Arbeitslohn vorschiessen. „Ces cas-là toutefois ne sont pas communs, et ${ }^{144}$ dans tous les pays de l'Europe, pour un ouvrier indépendant, il y en a vingt qui servent sous un maître ${ }^{145}$, et partout on entend, par salaires du travail, ce qu'ils sont communément quand l'ouvrier est une personne, et que le propriétaire du capital qui lui donne de l'emploi, en est une autre. ${ }^{146}$ C'est par la convention qui se fait habituellement entre ces deux personnes, dont l'intérêt n'est nullement le même ${ }^{147}$, que se détermine le taux commun des salaires. Les ouvriers desirent gagner le plus possible; les maîtres, donner le moins qu'ils peuvent. ${ }^{148}$ Avec cela il est aisé de prévoir lequel des deux partis, dans toutes les circonstances ordinaires, doit avoir l'avantage du débat, et forcer l'autre à céder à ses conditions. Les maîtres, étant en moindre nombre, peuvent non-seulement parlà se concerter plus aisement, mais la loi même les autorise à se concerter entr'eux, ou au moins ne le leur interdit pas, tandis qu'elle l'interdit aux ouvriers. Nous n'avons point d'actes du parlement contre les ligues qui tendent à abaisser le prix de l'ouvrage; mais nous en avons beaucoup contre celles qui tendent à le faire hausser. (p. 133) (l.c.) (cf. p. 134 l.c.) ${ }^{149}$

Wie viel mehr independent Arbeiter es selbst noch zur Zeit Smiths gab sieh p. 174, t. I ${ }^{150}$

$\mathrm{d}<\mathrm{d} .>$
e $<$ Arbeit $>$
$\mathrm{f}<\mathrm{Wa}>$
$\mathrm{g}<$ <einer bestim>

144 Bei Smith Übergang zu S. 133.

145 Drei Hervorhebungen Marxens.

146 Bei Smith Absatz.

147 Hervorhebung Marxens.

${ }^{148}$ Bei Smith folgt eine Aufzählung weiterer Interessengegensätze zwischen beiden Personengruppen, dann Absatz.

${ }^{149}$ S. 134: Les maitres sont en tout tems et partout dans une sorte de ligue tacite, mais constante et uniforme, pour ne pas élever les salaires au dessus du taux actuel. [...] A la vérité, nous n'entendons jamais parler de cette ligue, parce que c'est l'état habituel, et on peut dire l'état naturel de la chose, auquel personne ne fait attention.

${ }_{150}$ S. 174: D'ailleurs, une grande partie de l'ouvrage extraordinaire qui se fait probablement dans les années de bon marché, ne paraît jamais sur les registres publics des 
Accumulation. „la même cause qui fait hausser les salaires du travail, l'accroissement des capitaux ${ }^{151}$, tend à augmenter ses facultés productives, et tend à mettre une plus petite quantité de travail en état de produire une plus grande quantité d'ouvrage. (t. I, p. 177. 1. I ch. VIII. cf. p. 178 ${ }^{152}$ )

Gesetze gegen d. Arbeiter zu Gunsten d. maîtres. p. 296, 97. Schön dabei d. folgende Satz:

$/ 190 /$,Toutes les fois que la législature essaie de regler ${ }^{153}$ les démêlés entre les maîtres et leurs ouvriers, ce sont toujours les maîtres qu'elle consulte; u. ${ }^{154}$ quand le reglement ${ }^{155}$ est en faveur des ouvriers, il est toujours juste et raisonnable." (t. I p. 296)

Travail productif et non productif. 1. II. ch. III. t. II, p. 311. [(,,le travail d'un ouvrier de manufacture ajoute en général $\mathrm{a}^{156}$ a la valeur de la matière sur laquelle travaille cet ouvrier, la valeur de sa subsistance et du profit de son maître. ${ }^{157}$ - il ne lui coûte, dans le fait, aucune dépense"158 (t. II, p. 311)] p. 312. 313. 314. Capital tauscht sich nur direkt gegen $b$ travail productif aus [od. gegen andre Waaren] [nicht nur gegen Salaire] (aber d. Salair ist d. einzige Revenue, womit sich d. Capital im Productionsprocess austauscht:] Revenu gegen travail productif od. non productif. [D. Bestimmung, wenn auch falsch gefaßt, war tiefer bei d. Physiokraten daß nur d. Arbeit productif ist (im capit. Sinn) die surplus value schafft. Als solche betrachteten sie aber fälschlich - weil d. handgreiflichste Form - (u. weil sie Gebrauchswerth v. Tauschwerth nicht richtig scheiden) $-\mathrm{d}$. Agriculturarbeit.) Smith sagt:

„Cette partie du produit annuel de la terre et du travail d'un pays qui remplace un capital, n'est jamais immédiatement employée à entretenir d'autres salariés que des salariés productifs. ${ }^{159}$ elle ne paie ${ }^{160}$ des salaires

\footnotetext{
a $<$ la $>$

$b<$ cap $>$
}

manufactures. Les compagnons qui quittent leurs maîtres, se mettent ouvriers à leur propre compte. Les femmes retournent chez leurs parens, et ordinairement y filent pour faire des draps pour elles et pour leurs familles. Les ouvriers indépendans ne travaillent même pas toujours pour vendre au public, mais ils se trouvent employés par leurs voisins à des ouvrages de manufactures pour l'usage de la famille.

151 Hervorhebung Marxens.

152 Bei Smith finden sich auf S. 178 weitere Ausführungen zum Thema.

153 Bei Smith: régler

154 Bei Smith: aussi

155 Bei Smith: réglement

156 Bei Smith: à

157 Bei Smith: maître. [. . .] Quoique le premier reçoive des salaires que son maitre lui avance, il ne lui coûte

158 Hervorhebung Marxens.

159 Bei Smith: productifs;

160 Hervorhebung Marxens. 
qu'au travail productif seulement. Celle qui est destinée à former immédiatement un revenu, soit comme profit, soit comme rente, peut indifféremment entretenir des salariés productifs ou des salariés non productifs ${ }^{161}$. (351. t. II, 315. 1. II. ch. III) (cf. ib. p. 315, 316.)

Nach A. Smith selbst bleibt in d. That - m. Ausnahme dessen, daß travailleurs productifs solche sind, die an d. unmittelbaren Production v. Waaren arbeiten, im unmittelbaren Productionsproce $B$ - nur solche Arbeiter productif sind, die surplus value for their master, profit produciren. „Toute partie de ses fonds ' qu'un homme emploie comme capital, il s'attend toujours qu'elle lui rentrera avec un profit. Il ne l'emploie donc qu'à entretenir des salariés productif ${ }^{162}$; [aber ein Theaterentrepreneur wendet $d$. Schauspieler auch nur an, weil sie ihm Profit bringen, daher productive Arbeiter sind. Dépense sind sie f. d. Consummenten d. Theater, nicht für ihn. Dieß gilt aber auch vom Brod d. Bäcker.]; et après avoir fait, à son égard, office de capital, cette même partie de fonds forme un revenu à ses ${ }^{163}$ travailleurs. Toutes les fois qu'il emploie une partie de ces mêmes fonds à entretenir des salariés non-productifs de quelqu'espèce que ce soit, dès ce moment cette partie se trouve retirée de son capital et versée dans le fonds réservé $\mathrm{d}$ pour servir immédiatement à sa consommation. (p. 315 , 316)

Rente setzt A. Smith jetzt nur $1 / 3$ od. $1 / 4$ d. Products. À mésure ${ }^{164}$ des progrès que fait l'amélioration, la rente augmente bien relativement à l'étendue de la terre, mais elle diminue dans sa proportion avec le produit. "165 (t. II, p. 320) (1. II, ch. III) ditto m. d. Capitalprofit. (p. 320, 321. l.c.)

Accumulation. „la cause immédiate de l'augmentation du capital, c'est l'économie et non l'industrie. À la vérité, l'industrie fournit la matière $e^{166}$ des épargnes que fait l'économie; mais quelques gains que fasse l'industrie, sans l'économie qui les épargne et les amasse, le capital ne serait jamais plus grand. (t. II, p. 326. 1. II, ch. III) (Smith nimmt übrigens überall, sieh p. $326 s q q$ falsch an, daß im Verhältniß wie d. capital productif wächst, d. Anzahl d. ouvriers salariés, d. travailleurs /191/ productifs zunimmt.)

„Pour augmenter la valeur du produit annuel de la terre et du travail dans une nation, il n'y a pas d'autres moyens que d'augmenter, quant au nombre, les ouvriers productifs, ou d'augmenter, quant à la puissance, la

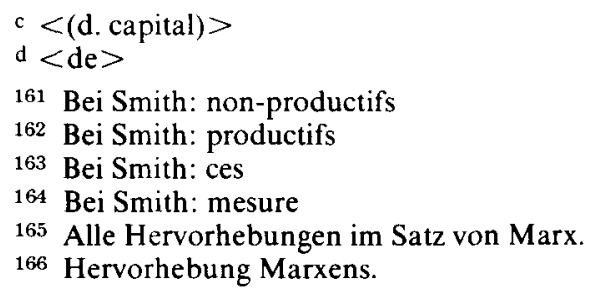


faculté productive des ouvriers précédemment employées ${ }^{167}$. In beiden Fällen surcroit de capital nöthig. (Sieh t. II p. 338, 339.)

,la concurrence des capitalistes fait hausser les salaires du travail et fait baisser les profits." (t. II p. 359)

1. I. ch. VI: „Comme dans un pays civilisé il n'y a que très peu de marchandises dont toute la valeur échangeable procède du travail seulement, et que, dans la très-majeu re partie d'entrea elles, la rente et le profit y contribuent pour des fortes portions ${ }^{168}$, il en résulte que le produit annuel $\mathrm{du}$ travail de ce pays suffira toujours pour acheter et commander une quantité de travail beaucoup plus grande que celle qu'il a fallu employer pour faire croître ce produit, le préparer et l'amener au marché. (p. 108, 109) (t. I)

\section{b Intérêt.p. 105. t. I. (stets secundär.)}

Gehört Profit zu d. Productionskosten od. nicht? Bei dieser Gelegenheit wirftc Smith d. Keim zu d. spätern „Consummationstheorie”. Er sagt:

1. I. ch. VII. (t. I, p. 111, 112): ,quoique, dans le langage ordinaire, quand on parle de ce qu'une marchandise coûte en première main, on n'y comprenne pas le profit de la personne qui fait métier de la vendre, cependant si celle-ci la vendait à un prix qui ne lui rendît pas son profit au taux ordinaire du canton, il est évident qu'elle perdrait à ce métier, puisqu'elle aurait pu faire ce profit en employant son capital d'une autre manière. ${ }^{169}$ (Hier um d. Profit in einer Anwendung d. Capitals zu erklären, vorausgesetzt, daß d er in andern Zweigen gemacht wird. Also e Profit vorausgesetzt um ihn zu erklären.) Aber nun kommts: „D'ailleurs, son profit constitue son revenu ${ }^{\mathbf{1 7 0}}$ (Profit also dadurch erklärt, daß Leute vom Profit als ihrer Revenue leben); c'est pour elle le fonds d'où elle tire f sa subsistance. (Dieß beweist, $\mathrm{da} ß$ es einen fonds unter $\mathrm{d}$. Namen Profit geben muß, woraus gewisse Leute ihre Subsistenz beziehn.) De même qu'elle avance à ses ouvriers leurs salaires ou leur subsistance pendant que la marchandise se prépare et est conduite au marché, de même cette personne s'avance aussi sa propre subsistance, ${ }^{171}$ qui en général est en raison du profit qu'elle peut raison-

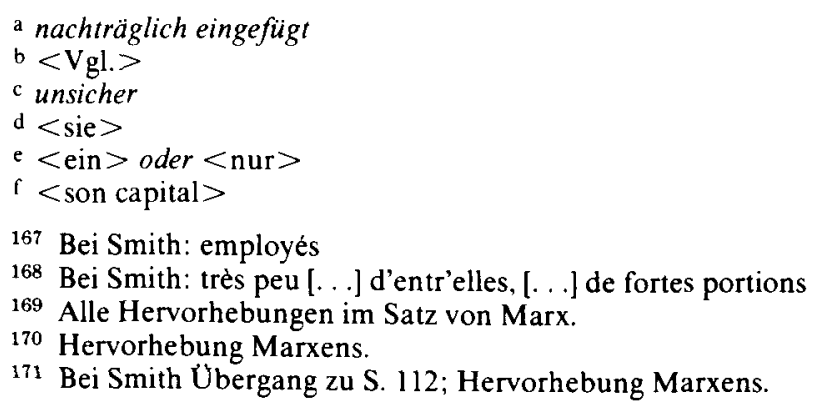


nablement attendre de la vente de sa marchandise. (Weil er als Capitalist, nicht als Arbeiter lebt, muß er als Capitalist bezahlt werden.) Ainsi, à moins de lui abandonner ce profit, on ne lui aura pas payé le prix qu'on peut regarder, à juste titre, comme celui que cette marchandise lui coûte réellement." $172 \mathrm{~S}$. Profit muß gezahlt werden, weil er ihn verfrißt. Aber beig A. Smith dieß nicht so albern, wie bei denen, die es zur /192/ Hauptraison d. Profits machen. Er a spricht in diesem Capitel nicht v. d. Entstehung d. Profits od. seiner Natur; sondern v. d. taux naturel unter d. Voraussetzung d. Profits schon als wesentlichem Motor d. Production, die er in d. That nur aus d. Trennung d. Klassen erklärt hat od. aus d. in d. Luft gesetzt sein d. ouvrier salarié.

/210/ ad p. 188. Die dort u. sqq. citirten Stellen v. A. Smith heissen englisch;

edit. v. McCulloch. London. 1828."'173

I. I. ch. VIII., The produce of labour constitutes the natural recompence or wages of labour. ${ }^{174}$ In that original state of things, which precedes ${ }^{175}$ both the appropriation of land and the accumulation of stock, the whole produce of labour belongs to the labourer. He has neither landlord nor master to share with him. ${ }^{176} \mathrm{Had}$ this state continued, the wages of labour would have augmented with all those improvements in its productive powers, to which the division of labour gives occasion. All things would gradually have become cheaper. They would have been produced by a smaller quantity of labour; and as the commodities produced by equal quantities of labour would naturally in this state of things be exchanged for one another, they would have been purchased likewise with the produce of a smaller quantity.” (vol. I, p. 107, 108) „But this original state of things, in which the labourer enjoyed the whole produce of his own labour, could not last beyond the first introduction of the appropriation of land and the accumulation of stock. It was at an end, therefore, long before the most considerablea improvements were made in the productive powers of

g nachträglich eingefügt

a $<$ will nur $>$

a co|:n:| siderable

172 Hervorhebung Marxens.

173 Adam Smith, An Inquiry into the Nature and Causes of the Wealth of Nations. With a Life of the Author, an introductory Discourse, Notes, and supplemental Dissertations.

By J. R. McCulloch, 4 Bde, Edinburgh, London 1828.

174 Bei Smith Absatz.

175 Bei Smith Übergang zu S. 108.

176 Bei Smith Absatz. 
labour, and it would be to no purpose to trace further what might have been its effects upon the recompence of ${ }^{177}$ wages of labour. ${ }^{178}$ As soon as land becomes private property, the landlord demands a share of almost all the produce which the labourer can eitherb raise, or collect from it. His rent makes the first deduction from the produce of the labour which is employed upon land. ${ }^{179}$ It seldom happens that the person who tills the ground has wherewithal to maintain himself till he reaps the harvest. His maintenance is generally advanced to him from the stock of a master, the farmer who employs him, and who would have no interest to employ him, unless he was to share in the produce of his labour, or unless his stock was to be replaced to him with a profit. This profit makes a second deduction from the produce of the labour which is employed upon land. ${ }^{180}$ The produce of almost all other labour is liable ${ }^{181}$ to the like deduction of profit. ${ }^{182}$ In all arts and manufactures the greater part of the workmen stand in need of a master to advance them the materials of their work, and their wages and maintenance till it be completed. He shares in the produce of their labour, or in the value which it adds to the materials upon which it is bestowed; and in this share consists his profit." (1.c. p. 109, 110) ${ }^{183}$

book II. ch. III. (vol. II) ,There is one sort of labour which adds to the value of the subject upon which it is bestowed; there is another which has no such effect. The former, as it produces a value, may be called productive, the latter ${ }^{184}$ unproductive labour. Thus the labour of a manufacturer adds generally to the value of the materials which he works upon, that of his own maintenance, and of his master's profit. The labour of a menial servant, on the contrary, adds to the value of nothing. Though the manufacturer has his wages advanced to him by his master, he in reality costs him no expence, the value of those wages being generally restored, together with a profit, in the improved value of the subject, ${ }^{185}$ upon which his labour is bestowed. But the maintenance of a menial servant never is restored. A /211/ man grows rich by employing a multitude of manufacturers; he grows poor by maintaining a multitude of menial servants. The labour of the latter, however, has its value, and deserves its reward as well as that of the former. But the

$\mathrm{b}$ nachträglich eingefügt

177 Bei Smith: or

178 Bei Smith Absatz.

179 Hervorhebung Marxens; bei Smith dann Absatz.

${ }_{180}$ Hervorhebung Marxens; bei Smith dann Absatz.

${ }^{181}$ Bei Smith Úbergang zu S. 110.

182 Hervorhebung Marxens.

183 Alle Hervorhebungen im Satz von Marx.

${ }^{184}$ Bei Smith Komma.

${ }^{185}$ Komma von Marx. 
labour of the manufacturer fixes and realizes itself in some particular subject or vendible commodity, which lasts for some time at least after that labour is past. It is, as it were, a certain quantity of labour stocked and stored up, to be employed, if necessary, upon some other occasion. That subject, or, what is the same thing, the price of that subject, can afterwards, if necessary, put into motion a quantity of labour equal to that which had originally produced it. The labour of the menial servant, on the contrary, does not fix or realize itself in any particular subject or vendible commodity. His services generally perish in the very instant of their performance, and seldom leave any trace or value behind them, for which an equal quantity of service could afterwards be procured. (p. 93, 94 1.c. $)^{186}$

That part of the annual produce of the land and labour of any country which replaces a capital, never is immediately employed to maintain any but productive hands. ${ }^{187}$ It pays the wages of productive labour only. That which immediately ${ }^{188}$ destined for constituting a revenue, either as profit or as rent, may maintain indifferently either productive or unproductive hands. (p. 98) „Whatever part of his stock a man employs as a capital, he always expects it to be replaced to him with a profit. He employs it, therefore, in maintaining productive hands only; and after having served in the function of capital to him, it constitutes a revenue to them. Whenever he employs any part of it in maintaining unproductive hands of any kind, that part is from that moment withdrawn from his capital, and placed in his stock reserved for immediate consumption." (l.c.) If ${ }^{189}$ the quantity of food and clothing which were .. consumed ${ }^{190}$ by unproductive, had been distributed among productive hands, they would have reproduced, together with a profit, the full value of their consumption. (p. 109 l.c.) $/ 212 /$ "The labour of some of the most respectable orders in the society is, like that of menial servants, unproductive of any value, and does not fix and realise $^{\mathbf{1 9 1}}$ itself in a any permanent subject, or vendible commodity ... The sovereign, for example, with all the officers both of justice 192 and war who

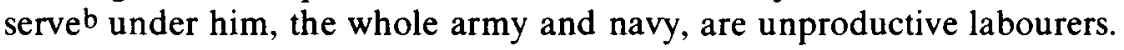
They are the servants of the public, and are maintained by a part of the annual produce of the industry of other people.... In the same class must

a nachträglich eingefügt

b se | :r: | ve

86 Alle Hervorhebungen im Abschnitt von Marx.

.87 Alle Hervorhebungen im Satz von Marx.

${ }_{88}$ Hervorhebung Marxens; bei Smith: That which is immediately

89 Bei Smith: But if

.90 Bei Smith: which were thus consumed

91 Bei Smith: or realize

92 Bei Smith Übergang zu S. 95. 
be ranked ... churchmen, lawyers, physicians, men of letters of all kind ${ }^{193}$; players, buffoons, musicians, opera-singers, opera-dancers etc. ${ }^{194}(94,95$ l.c.)

It is the highest impertinence and presumptionc, therefore, in kings and ministers, to pretend to watch over the economy of private people, and to restrain their expense, either by sumptuary laws or by prohibiting the importation of foreign luxuries. They are themselves always, and without any exception, the greatest spendthrifts in the society. ${ }^{195}$ Let them look well after their own expense, and they may safely trust private people with theirs. If their own extravagance does not ruin the state, that of their subjects never will. (p. 121, 122 ${ }^{196}$ ) (l.c.)

I. II. ch. IV. (v. II) (Interest)

The stock which is lent at interest is always considered as a capital by the lender. He expects that in due time it is to be restored to him, and that in the mean time the borrower is to pay him a certain annual rent for the use of it. The borrower may use it either as a capital, or as a stock reserved for immediate consumption. If he uses it as a capital, he employs it in the maintenance of productive labourers, who reproduce the value with a profit. He can, in this case, both restore the capital and pay the interest, without alienating or encroaching upon any other source of revenue. If he uses it as a stock reserved for immediate consumption, he acts the part of a prodigal, $/ 213 /$ and dissipates, in the maintenance of the idle, what was destined for the support of the industrious. He can, in this case, neither restore the capital nor pay the interest, without either alienating or encroaching upon some other source of revenue, such as the property or the rent of land. $(127)^{197}$

I volume D. elenden MacCulloch "Introductory discourse”, dasselbe, was dieser Hund of ,the wages of accumulated labour" als discourse über d. Geschichte d. pol. Oekonomie hat drucken lassen. (Vorher)

Introductory Discourse (v. d. elenden MacCulloch, t. I) Darin citirt:

Petty in his tract: a ,On Taxes and Contributions" $(1667)^{198}$ :

"If a man bring to London an ounce of silver out of the earth in Peru, in the same time that he can produce a bushel of corn, the one is the natural price

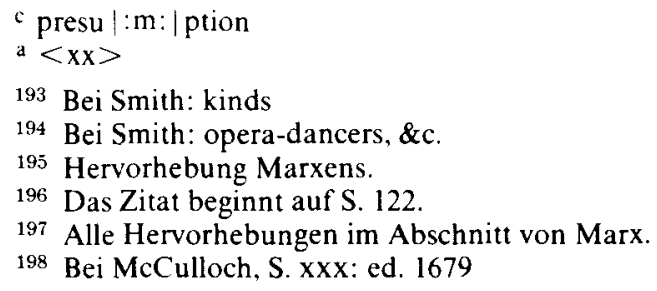


of the other; now, if, by reason of new and more easier ${ }^{199}$ mines, a man can get two ounces of silver as easily as formerly he did one, then corn will be as cheap at $10^{200}$ shillings the bushel as it was before at $5 \mathrm{sh}^{201}$ caeteris paribus." 202 „Let a hundred men work $10^{203}$ years upon corn, and the same number of men the same time upon silver; I say that the neat proceed of the silver is the price of the whole neat proceed of the corn; and like parts of the one the price of like parts of the other." „Corn will be twice as dear when there are $200^{204}$ husbandmen to do the same work which $b$ an hundred could perform." 205 (ed. 1679. p. 31, 24, 67)

Considerations on the East India Trade. 1701. (p. 51, 52 sqq) ${ }^{206}$

„The East India trade destroys no profitable English manufacture; it deprives the people of no employment which we should wish to be preserved. The foundation of this complaint is that manufactures are procured from the East Indies by the labour of fewer people than are necessary to make the same in England; and this shall be admitted. Hence $/ 214 /$ it follows that to reject the Indian manufactures that like may be made by the labour of more hands in England, is to employ many to do the work that may be done as well by few; is to employ all, more than are necessary to procure such things from the East Indies, to do work that may be done as well without them. ${ }^{207}$ A saw mill with a pair or two of hands, will split as many boards as thirty men without this mill; if then the use of this mill shall be rejected, that $30^{208}$ may be employed to do the work, $28^{209}$ a are employed more than are necessary, or are employed to do ab work that may be as well done without them. So if by any art, or trade, or engine, the labour of one can produce as much for our consumption, or other use, as can otherwise be procured only by the labour of 2 or $3^{210}$; if this art, or trade, or engine, shall be rejected, if $3^{211}$ shall be employed to do the work

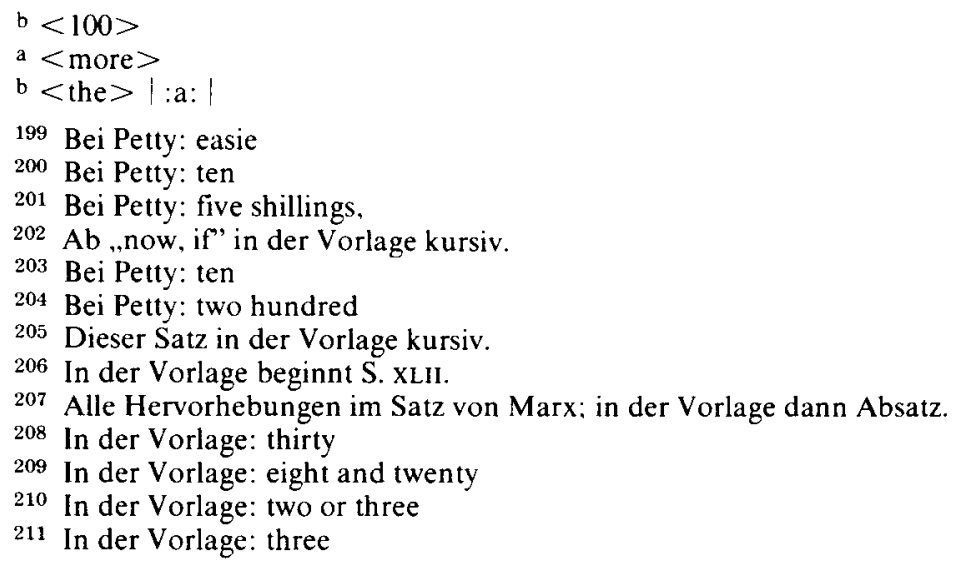


of one, two are employed more than necessary ${ }^{212}$, or to profit of the kingdom. For if the providence of god ${ }^{213}$ should provide corn for England as manna heretofore for Israel, the people would not be well employed to plough, and sow, and reap, for no ${ }^{214}$ more corn than might be made 215 without this labour. Wherefore to employ more hands to manufacture things in England than are necessary to procure the like from India, is to employ so many to no profit that might otherwise be profitably employed. For there can be no want of profitable employment so long as England is not built, beautified, and improved to the utmost perfection; so long as we either have or can produce any thing that others want, or that they have any thing that we want. ${ }^{216}$ We are very fond of being restrained to the consumption of English manufactures, and, therefore, contrive laws either directly or by high customs, to prohibit all that come from foreign countries. By this time 'tis easy to see some of the natural consequences of this prohibition: $-{ }^{217}$ 'Tisc to oblige things to be provided by the labour of many, which might as well be done by few; 'tis to oblige many to labour to no purpose, to no profit of the kingdom, nay, to throw /215/ away their labour which otherwise might be profitable. ${ }^{218}$ 'Tis all one as to bid us to refuse bread or clothes, though the providence of $\operatorname{god}^{219}$ or bounty of our neighbours should bestow them on us; 'tis all one as to destroy an engine or navigable river, that the work which is done by few may be done by many. ${ }^{220}$ As often as I consider these things, I am ready to say with myself, that god $^{221}$ has bestowed his blessings upon men that have neither hearts ${ }^{222}$ nor skill to use them. For, why are we surrounded with the sea? Surely that our wants at home might be supplied by our navigation into other countries. By this we taste the spices of Arabia, yet never feel the scorching sun that brings them forth; we shine in silks which our hands have never wroughta; we drink of vineyards which we never planted; the treasures of these ${ }^{223}$

$c<.,>$ Tis

a wrougth

212 In der Vorlage: than are necessary

213 In der Vorlage: God

${ }^{214}$ In der Vorlage Übergang zu S. XLIII.

215 In der Vorlage: had

216 In der Vorlage Absatz.

217 In der Vorlage Absatz.

${ }^{218}$ Marx überschlägt folgenden Satz: 'Tis to provide the conveniences of life at the dearest and most expensive rates, to labour for things that might be had without.

${ }^{219}$ In der Vorlage: God

220 In der Vorlage Absatz.

221 In der Vorlage: God

${ }_{222}$ In der Vorlage Übergang zu S. xLIV.

223 In der Vorlage: those 
$\mathrm{b}$ mines are ours, in which we have never digged; we only plough the deep and reap the harvest of every country in the world."

In $d$. IV Band (diese Ausgabe d. A. Smith) ${ }^{\mathfrak{c}}$ d. notes u. dissertations d. elenden McCulloch:

"Labour may properly be defined to be any sort of action or operation, whether performed by man, the lower animals, machinery, or natural agents, that tends to bring about any desirable result. (p. 75) 224 ,In so far, however, as that result is effected by the labour or operation of natural agents, that can neither be monopolized nor appropriated by a greater or smaller number of individuals to the exclusion of others, it has no value. What is done by these agents is done gratuitously. (l.c.) „If a capitalist expends the same sum in paying the ${ }^{d}$ wages of labourers, in maintaining horses, or in hiring a machine, and if the men, the horses, and the machine can all perform the same piece of work, its value will obviously be the same by whichever of them it may be ${ }^{225}$ performed. (p. 77) When ${ }^{226}$ it is said that commodities are in all cases e wholly produced by means of labour, reference is necessarily made to all sorts of labour, whether of man, capital, or natural agents. But when it is said, that the value of the commodity or product is determined by the quantity of labour expended in its production, reference is made only to that species of labour which is possessed of value, that is, to the labour of man, $o r^{227}$ of capital expended upon the commodity or product. (76) „There are a variety of products which, after they are brought into a certain state, and are no longer subjected to the immediate and direct action of man or machinery, notwithstanding undergo further changes, and in consequence acquire additional value. Now the question is ${ }^{228}$ of what are these changes and the additional ${ }^{229}$ value the result? Those who contend that the increased value is not the result /216/ of labour, say that it is a compensation for the time the capital invested in the commodities is retained in a state of inaction. But that this cannot possibly be the case, is obvious from the circumstance, that were no

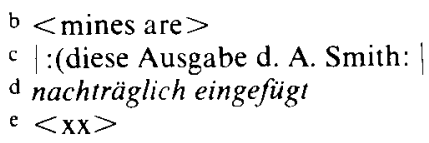

224 Bei McCulloch: It appears, therefore, that labour may properly be defined to be any sort of action or operation, whether performed by man, the lower animals, machinery, or natural agents, that tends to bring about any desirable result.

225 Bei McCulloch: have been

226 Bei McCulloch: And, therefore, when

227 Hervorhebung Marxens.

228 Bei McCulloch: is, of

229 Bei McCulloch: this increased 
change a produced upon the commodities, they might be retained in a cellar or a warehouse for the most lengthened period, without acquiring the smallest additional value. It follows, therefore, that the change that has been effected is the proximate and immediate cause of the additional value acquired by ${ }^{230}$ commodities; and as time can of itself produce no change or effect, and is only, if I may so speak, the space wherein really efficient agents operate, it seems abundantly clear that it can have nothing to do with the matter. (78)

There is not, indeed, a shade of difference, in point of principle, between the processes of fermentation etc, ${ }^{231}$ carried on inside a cask of wine or beer, and the action of the wind on the sails of a ship, or the blades of a wind-mill. And if we are justified in saying that corn is ground by means of labour, we must also be justified in saying that ${ }^{b}$ wine or beer is purified by means of labour. $A^{c}$ cask of new wine is a capital which represents, or is the result of the labour expended in cultivating, gathering, pressing etc ${ }^{232}$ the grapes of which it is made; and the action or labour of fermentation, and of the other processes carried on inside the cask, has precisely the same effect on this capital that the action or labour of the wind has on the capital invested in a ship or a mill. It produces the desired change or effect; but the value of that change is not increased by, and is in no degree dependent on, the operation or labour of the natural agents concerned, but on the amount of capital, or the produce of previous labour, that co-operated in the production of the effect; just as the cost of grinding corn does not depend on the action of the wind or water that turns the mill, but on the amount of capital wasted in $/ 217 /$ that $^{233}$ operation. (79) The word labour means here, and in all discussions respecting value, ... either the immediate labour of men, or the labour of the capital produced by man, or both. (p. 84 1.c.) $)^{234}$

It is necessary ${ }^{235}$ to distinguish between the exchangeable value, and the real or cost value of commodities or products. By the first, or the ex-

a cha $\mid$ :n: |ge

b th $|: a:| t$

c $A<s>$

230 Bei McCulloch: by the

231 Bei McCulloch:, \&c.

232 Bei McCulloch: pressing, \&c.

233 Bei McCulloch: of the capital wasted in the

234 Bei McCulloch: It is, perhaps, unnecessary to remark, that the word labour means here, and in all discussions respecting value, unless the contrary be distinctly mentioned, - either the immediate labour of man, or the labour of the capital produced by man, or both; as was explained in the note on Labour.

235 Bei McCulloch: And hence, in order to disentangle this important subject, and to set it in a clear point of view, it is necessary 
changeable value of a commodity or product, is meanta its power or capacity of exchanging either ${ }^{236}$ for other commodities or for labour; and by the second, or its real or cost value, is meant the quantity of labour which is required for its production or appropriation, or rather the quantity which would be required for the production or appropriation of a similar commodity at the time when the investigation is made. $(85,86)$

A commodity produced by a certain quantity of labour will [when the supply of commodities is equal to the effectual demand] uniformly exchange for, or buy any other commodity produced by the same quantity of labour. It will never, however, exchange for, or buy exactly the same quantity of labour that produced it; but though it will ${ }^{237}$ not do this, it will always exchange for, or buy the same quantity of labour as any other commodity produced under the same circumstances, or by means of the same quantity of labour, as itself. ${ }^{238}(96,97)$ In point of fact, it (the commodity) will always exchange for more [viz. more labour than that by which it was produced]; and it is this excess that constitutes profits. No capitalist would have any motive to exchange the produce of a given quantity of labour already performed for the produce of the same quantity of labour to be performed. This would be to lend without receiving any interest on the loan. $(96$, Note)

a me $: a: \mid n t$

236 Bei McCulloch Übergang zu S. 86.

237 Bei McCulloch Übergang zu S. 97.

238 Bei McCulloch ab ..the same quantity of labour" kursiv. 